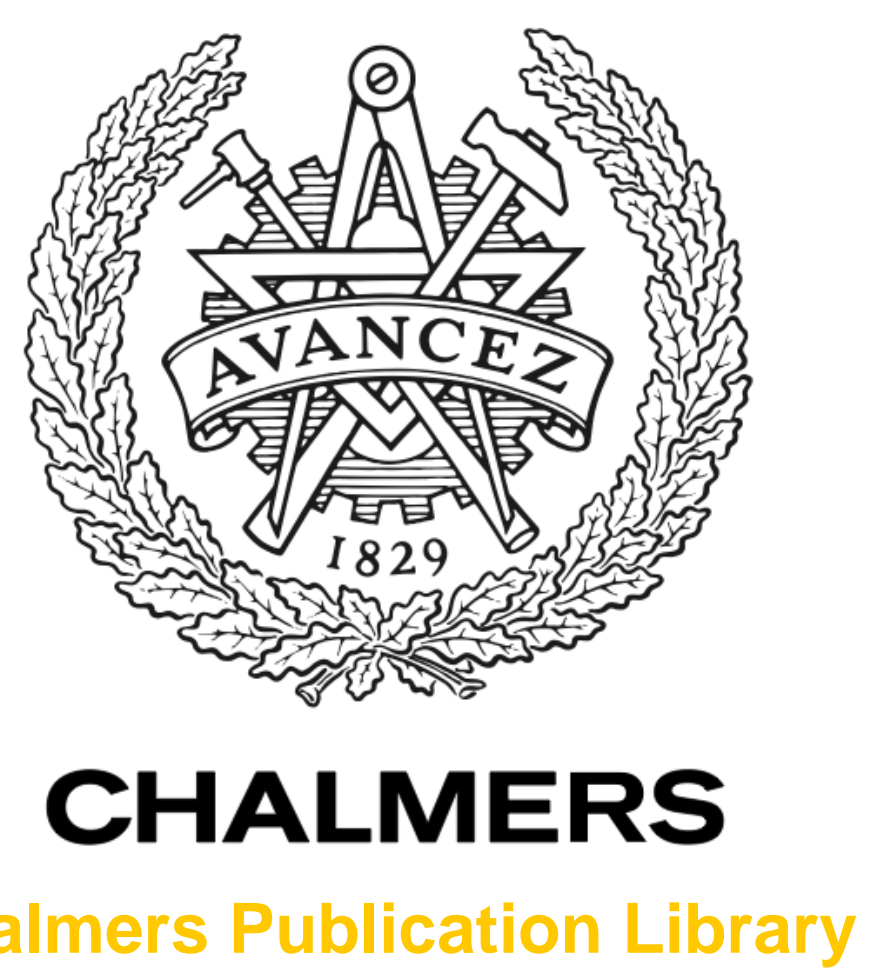

\title{
Radio Resource Management for D2D-based V2V Communication
}

This document has been downloaded from Chalmers Publication Library (CPL). It is the author's version of a work that was accepted for publication in:

IEEE Transactions on Vehicular Technology (ISSN: 0018-9545)

Citation for the published paper:

Wanlu, S. ; Ström, E. ; Brännström, F. et al. (2015) "Radio Resource Management for D2Dbased V2V Communication". IEEE Transactions on Vehicular Technology

Downloaded from: http://publications.lib.chalmers.se/publication/222774

Notice: Changes introduced as a result of publishing processes such as copy-editing and formatting may not be reflected in this document. For a definitive version of this work, please refer to the published source. Please note that access to the published version might require a subscription. 


\title{
Radio Resource Management for D2D-based V2V Communication
}

\author{
Wanlu Sun, Erik G. Ström, Fredrik Brännström, Kin Cheong Sou, and Yutao Sui
}

\begin{abstract}
Direct device-to-device (D2D) links have been proposed as a possible enabler for vehicle-to-vehicle (V2V) communications, where the incurred intra-cell interference and the stringent latency and reliability requirements are challenging issues. In this paper, we investigate the radio resource management problem for D2D-based V2V communication. Firstly, we analyze and transform the latency and reliability requirements of $\mathrm{V} 2 \mathrm{~V}$ communication into optimization constraints that are computable using only the slowly varying channel information. This transformation opens the possibility of extending certain existing D2D techniques to cater for V2V communication. Secondly, we propose a problem formulation that fulfills the different requirements of $\mathrm{V} 2 \mathrm{~V}$ communication and traditional cellular communication. Moreover, a Separate resOurce bLock and powEr allocation (SOLEN) algorithm is proposed to solve this problem. Finally, simulations are presented to evaluate different schemes, which illustrate the necessity of careful design when extending D2D methods to V2V communication and also show promising performance of the proposed SOLEN algorithm.
\end{abstract}

\section{INTRODUCTION}

\section{A. Motivation}

Recently, vehicle-to-vehicle (V2V) communications have attracted great interest due to the potential to improve traffic safety, reduce energy consumption, and enable new services related to intelligent transportation systems. Usually, these types of applications have a strongly localized nature, i.e., requiring cooperation between vehicles in close proximity. Furthermore, other common features to most applications are real-time requirements, as well as strict requirements on reliability and access availability. For instance, the EU project METIS considers that a maximum end-to-end latency of $5 \mathrm{~ms}$, with transmission reliability of $99.999 \%$ of 1600 bytes packets should be guaranteed to deliver traffic safety and efficiency applications [1].

This work has been supported in part by the Swedish Research Council project 2011-5824. Part of this work has been performed in the framework of the FP7 project ICT-317669 METIS, which is partly funded by the EU. The authors would like to acknowledge the contributions of their colleagues in METIS. The calculations were performed on resources provided by the Swedish National Infrastructure for Computing (SNIC) at C3SE.

Copyright (c) 2015 IEEE. Personal use of this material is permitted. However, permission to use this material for any other purposes must be obtained from the IEEE by sending a request to pubs-permissions@ieee.org.

Wanlu Sun, Erik G. Ström, Fredrik Brännström, Kin Cheong Sou, and Yutao Sui are with the Department of Signals and Systems, Chalmers University of Technology, Gothenburg, Sweden (e-mail: wanlu@chalmers.se; erik.strom@chalmers.se; fredrik.brannstrom@chalmers.se; kincheong.sou@chalmers.se; sui.yutao@chalmers.se).
Current legacy solutions for $\mathrm{V} 2 \mathrm{~V}$ communication are adhoc communications over the 802.11p standard and backendbased communications over the Long Term Evolution (LTE) cellular standard. The main problem with the 802.11 p legacy system is that the physical (PHY) layer is regular 802.11 OFDM with $10 \mathrm{MHz}$ channel spacing option and that the medium access control (MAC) layer is regular 802.11 carrier sense multiple access (CSMA). As such, these are mainly optimized for a WLAN-type of environment with no or very slow mobility, and thus not optimized for vehicles with high mobility. Furthermore, the lack of stringent quality of service (QoS) provisioning and centralized management in the 802.11p standard challenges the fulfilment of the requirements on V2V applications as well. On the other hand, in LTE systems, as analyzed in [2], the performance for vehicular communication is not always satisfactory, especially in terms of latency and reliability. Therefore, there is a strong desire of finding better solutions to support V2V communication.

Device-to-device (D2D) communication is identified as one of the technology components for future cellular systems. For example, the third generation partnership project (3GPP) agrees that D2D communication will become one of the new features to be studied during 3GPP Rel-12 and Rel-13 timeframes [3]. Also, the METIS project regards D2D as one of the horizontal topics which are the main technical solutions in the fifth generation (5G) networks [1]. In a D2D underlaying cellular infrastructure, two physically close user equipment (UE) devices can directly communicate with each other by sharing the same resources used by regular cellular UEs (CUEs). Correspondingly, three promising gains, i.e., proximity gain, reuse gain, and hop gain, may be offered [4].

By comparing the QoS requirements of V2V communication and the potential benefits of D2D communication, it turns out that the direct D2D link can be a possible enabler for V2V communication due to the following reasons. Firstly, the localized nature of $\mathrm{V} 2 \mathrm{~V}$ services is exactly the motivating idea for D2D communication. Moreover, the low latency requirement of $\mathrm{V} 2 \mathrm{~V}$ applications fits the hop gain of D2D transmissions. Last but not least, V2V's requirement on high reliability is consistent with the proximity gain provided by D2D links. Nevertheless, using D2D underlay for V2V communication, if performed blindly, may cause significant degradation to system performance due to the interference introduced by resource reuse. Also, to guarantee the required latency and reliability is still a challenge that needs to be tackled for V2V services. Hence, radio resource management (RRM) becomes a key 
design aspect to enable D2D-based V2V communication.

\section{B. State of the Art}

Even though the D2D underlay network is an appealing solution for $\mathrm{V} 2 \mathrm{~V}$ communication, to the best of our knowledge, only a few studies [1], [5]-[8] have been conducted along this direction. The suitability of D2D techniques to V2V applications was systematically discussed in [1], [5], [6]. The authors in [7] proposed a heuristic location dependent resource allocation scheme to protect vehicular users. The performance of C-UEs, however, is not optimized. Besides, we have in [8] proposed a two-stage RB allocation and power control scheme SRBP for D2D-based V2V communication when taking into account the QoS requirements of both vehicular UEs (V-UEs) and C-UEs.

On the other hand, extensive researches have been carried out in the context of traditional D2D systems, where one of the most critical challenges is the interference between the primary cellular network and the D2D underlay. To cope with this new interference situation, one crucial issue is the RRM strategy, which includes how C-UEs and potential D2D UEs choose the resources to share, and how each UE allocates its transmit power among its used resources. There have been many efforts investigating the RRM problem in such a system. Due to the space limitation, we will only name a few in this field. The interested readers can find more information in the excellent survey papers [4], [9]-[11], and the references therein.

To maximize the sum rate of the whole network, the authors in [12]-[18] proposed various algorithms. The work in [13] presented mode selection and power control scheme for one D2D link and one C-UE. To generalize the system model, [12] studied the resource allocation for multiple D2D links and C-UEs. The authors in [14] increased the sum rate by avoiding the near-far interference from C-UEs to D2D links. Recently, more advanced mathematical techniques have been exploited in RRM problems. An iterative combinatorial auction game was utilized in [15] to derive a spectrum resource allocation mechanism. Also, a near optimal resource sharing algorithm was proposed in [17] by formulating the interference relationships among different D2D and cellular links as an interference-aware graph. Particularly, a sophisticated threestep resource allocation and power control scheme was derived in [16] to maximize the sum rate while guaranteeing the signal to interference plus noise ratio (SINR) requirements for both D2D users and C-UEs, which showed promising performance.

Nevertheless, none of the above D2D approaches can be directly applied to $\mathrm{V} 2 \mathrm{~V}$ communication, which has strict requirements on latency and reliability, due to the following limitations.

Firstly, most existing work aim to maximize the sum rate and prioritize cellular links. Whereas, the D2D underlay is only considered as opportunistic when their interference to the cellular links is controlled at acceptable levels.

Secondly, most papers interpret the QoS requirement directly from the SINR viewpoint, i.e., the achieved SINR should be above a particular target value. However, it is not straightforward and clear that how to obtain this target value from the original requirements of $\mathrm{V} 2 \mathrm{~V}$ communication, which usually refer to the reliable transmission of a certain amount of data within certain frequency bandwidth and time period [1].

Last but not least, the majority of the literature assume that the eNB is aware of the full instantaneous channel state information (CSI) of all the cellular and D2D links, which might be possible when the D2D users are static or slowly moving. However, this assumption is too optimistic for fast moving D2D-based V2V communication, where the vehicle related channels change rapidly.

\section{Contributions}

The problem under study is centralized RRM for D2Dbased V2V communication with strict latency and reliability requirements and with access only to slowly time-varying CSI. From now on, we denote the D2D-based V2V users as V-UEs. The main contributions are

- A method to transform the latency and reliability requirements of $\mathrm{V} 2 \mathrm{~V}$ communication into optimization constraints that are computable with only slowly varying CSI. The method allows us to extend certain existing D2D RRM algorithms, e.g., [12]-[17], to cater also for $\mathrm{V} 2 \mathrm{~V}$ communication with strict latency and reliability requirements and still maintain good performance. In contrast, a naive modification of existing algorithms performs poorly.

- An RRM problem formulation for allocating multiple RBs and transmit power to a set of C-UEs and VUEs. The problem is stated as an optimization problem with the objective to maximize the C-UE sum rate with proportional bandwidth fairness under the constraint of satisfying the V-UEs' requirements on latency and reliability.

- A heuristic method to approximately solving the RRM optimization problem with reduced complexity, but still with very promising performance.

\section{SySTEM MODEL}

\section{A. System Model}

Sets are denoted by calligraphic letters $\mathcal{X}$ and their cardinalities are denoted by $|\mathcal{X}|$. Besides, $\mathbb{E}[\cdot]$ indicates the expectation.

We consider a single cell environment where $M^{\prime} \mathrm{C}$-UEs and $K^{\prime}$ V-UEs (counted in terms of transmitters) share the available uplink radio resources, and the D2D underlay is

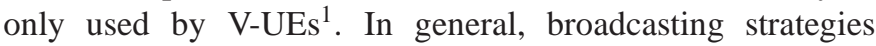
are used for vehicular communication. In this paper, we consider the least favorable receiving vehicle inside the intended broadcast region of each transmitting vehicle, i.e., the vehicle that has the smallest average channel gain from the transmitting vehicle. The user sets for C-UEs and V-UEs are $\mathcal{M}^{\prime} \triangleq\left\{1,2, \ldots, M^{\prime}\right\}$ and $\mathcal{K}^{\prime} \triangleq\left\{1,2, \ldots, K^{\prime}\right\}$, respectively.

\footnotetext{
${ }^{1}$ The extension to the coexistence of both traditional D2D UEs and V-UEs is left for future work.
} 


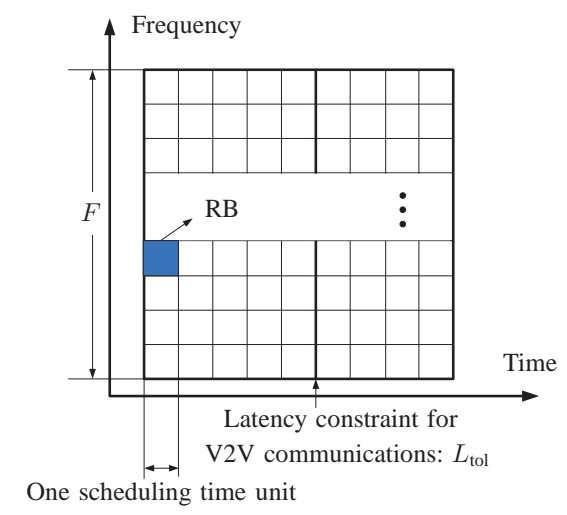

Figure 1. Two dimensional RBs for RRM.

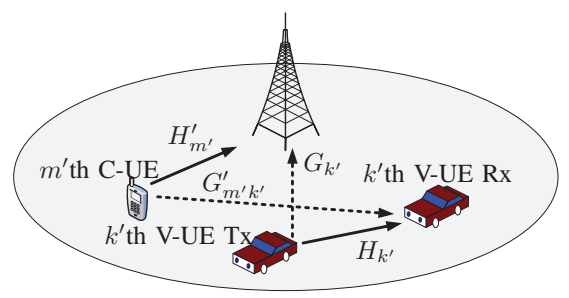

Figure 2. Interference between $\mathrm{V} 2 \mathrm{~V}$ and cellular communications

In our study, the available resources are two dimensional, i.e., including both frequency and time domains, where the whole uplink frequency bandwidth is divided into $F$ subbands, with $\mathcal{F} \triangleq\{1,2, \ldots, F\}$, for each transmission time unit, as illustrated in Fig. 1. Besides, one subband over one scheduling time unit is defined as one resource block (RB). The C-UEs use orthogonal RBs to communicate with the eNB, and the V-UEs use orthogonal RBs among each other. However, an $\mathrm{RB}$ can be used by both a C-UE and a V-UE. In this way, interference between the $\mathrm{V} 2 \mathrm{~V}$ and cellular transmissions will occur.

Fig. 2 illustrates the interference situation. Assume the $m^{\prime}$ th $\mathrm{C}$-UE and the $k^{\prime}$ th V-UE are using the same RB. Then they will cause intra-cell interference to each other. $H_{m^{\prime}}^{\prime}$ and $H_{k^{\prime}}$ are the channel power gains of the desired transmissions for the $m^{\prime}$ th C-UE and the $k^{\prime}$ th V-UE, respectively. $G_{m^{\prime} k^{\prime}}$ denotes the power gains of the interference channel from the $m^{\prime}$ th C-UE to the $k^{\prime}$ th $\mathrm{V}$-UE receiver, and $G_{k^{\prime}}^{\prime}$ represents the interference channel power gain from the $k^{\prime}$ th $\mathrm{V}$-UE to the eNB. To perform RRM, the eNB needs CSI (at least with certain level) for all these involved links, where $H_{m^{\prime}}^{\prime}$ and $G_{k^{\prime}}^{\prime}$ can be measured at the eNB itself, but $H_{k^{\prime}}$ and $G_{m^{\prime} k^{\prime}}$ have to be measured at the corresponding V-UE receiver and then reported back to the eNB. All channel power gains include path loss and shadowing fading, but ignore small scale fading. For this reason, we will call $\left\{H_{m^{\prime}}^{\prime}, H_{k^{\prime}}, G_{m^{\prime} k^{\prime}}, G_{k^{\prime}}^{\prime}\right\}$, for $m^{\prime} \in \mathcal{M}^{\prime}$ and $k^{\prime} \in \mathcal{K}^{\prime}$, for the slow CSI.

\section{B. Time Scale and Channel Acquisition for RRM}

Besides proximity gain, reuse gain, and hop gain, another potential advantage of D2D communication is to offload the eNB scheduler [4]. To indeed achieve this offloading gain, the time scale of interactions between the eNB and D2D UEs should be much longer than the traditional LTE scheduling time interval (1 ms). Furthermore, when D2D communication is used for $\mathrm{V} 2 \mathrm{~V}$ services, the channels related to V-UEs could change very fast. In this case, if the eNB wants meaningful short-term RRM, such as every millisecond, the V-UEs need to report their channels (i.e., $H_{k^{\prime}}$ and $G_{m^{\prime} k^{\prime}}$ ) every millisecond, which will cause potentially large overheads. For these two reasons, we claim that the eNB should do long-term, e.g., a few hundred milliseconds, RRM for D2D-based V2V communication. Long-term RRM can also be beneficial for V-UEs that are temporarily out of coverage, as it guarantees resources for these V-UEs.

Regarding channels related to V-UEs, during the considered long-term time period, slow fading effects including path loss and shadowing are quite similar and correlated, but the small scale fading (SSF) changes very fast due to high mobility. Therefore, the available channel information at the eNB should only take the slow fading effects into account since the RRM results must be valid for the next few hundred milliseconds. In this way, the V-UEs merely need to report the slow fading related channel information to the eNB every few hundred milliseconds, which gives a fairly low signaling overhead. Since path loss and shadowing components are not greatly influenced by the RB index $f$, the slow CSI in Section II-A is assumed to be independent of $f$.

\section{REQUIREMENTS ON V-UES AND C-UES}

Usually, V-UEs and traditional C-UEs usually have different types of interests. Hence, their QoS requirements should be different. In this section, we will clarify what our real goal is, and mathematically formulate the requirements of both V-UEs and C-UEs.

\section{A. Requirements of $V$-UEs}

V2V services usually have stringent latency and reliability requirements but are less interested in high data rate. Hence, their requirements can be modeled as strict constraints in our formulation. Now we will study how to consider these requirements in a mathematical way.

Due to the latency constraints in V2V communication, the RBs assigned to each V-UE should be contained in a limited time span. Besides, the considered frequency bandwidth is also limited. Hence, the number of RBs that are used for each V-UE's transmission is limited. As analyzed in [19], when assuming a finite number of RBs, $E_{k^{\prime}}^{\text {all }}$, for the $k^{\prime}$ th V-UE's transmission, the outage probability evaluated at a required number of bits $N_{k^{\prime}}$ is defined as

$$
p_{k^{\prime}}^{\text {out }} \triangleq \operatorname{Pr}\left\{\sum_{i=1}^{E_{k^{\prime}}^{\text {all }}} \rho \log _{2}\left(1+\gamma_{i}\right)<N_{k^{\prime}}\right\},
$$

where $\rho$ is the number of complex symbols per RB, $\gamma_{i} \triangleq$ $\bar{P}_{i}^{\mathrm{r}}\left|h_{i}\right|^{2} /\left(\sigma^{2}+\bar{S}_{i}^{\mathrm{r}}\left|g_{i}\right|^{2}\right)$ is the instantaneous SINR on the $i$ th $\mathrm{RB} ; \bar{P}_{i}^{\mathrm{r}}$ and $\bar{S}_{i}^{\mathrm{r}}$ are average received power from the desired and interfering users, respectively; $h_{i}$ and $g_{i}$ are random variables which represent the SSF effects of the desired 
channel and interference channel respectively; and $\sigma^{2}$ is the noise power. Then, similar to [1], the reliability requirement is interpreted from the perspective of outage probability and can be expressed as

$$
p_{k^{\prime}}^{\text {out }} \leq p_{\mathrm{o}}
$$

where $p_{\mathrm{o}}$ is the maximum tolerable outage probability.

Furthermore, as explained in Section II-B, the eNB only has knowledge of the slow fading effects of the channels. In this case, the reliability constraint considered by the eNB for implementing RRM should only involve the slow fading information. To achieve this, we will replace the requirement in (2) by a more strict requirement. We first upper-bound $p_{k^{\prime}}^{\text {out }}$ via the following Lemma 1.

Lemma 1. $p_{k^{\prime}}^{\text {out }}$ is upper-bounded by

$p_{k^{\prime}}^{\text {out }} \leq \operatorname{Pr}\left\{\sum_{i=1}^{E_{k^{\prime}}^{\text {all }}} \rho \log _{2}\left(1+\bar{\gamma}_{i}\left|h_{i}\right|^{2} \min \left\{\frac{1}{\left|g_{i}\right|^{2}}, 1\right\}\right)<N_{k^{\prime}}\right\}$,

where $\bar{\gamma}_{i} \triangleq \bar{P}_{i}^{r} /\left(\sigma^{2}+\bar{S}_{i}^{r}\right)$ only includes slow $C S I^{2}$.

Proof: First we notice that

$$
\gamma_{i}=\bar{\gamma}_{i}\left|h_{i}\right|^{2} \frac{\sigma^{2}+\bar{S}_{i}^{\mathrm{r}}}{\sigma^{2}+\bar{S}_{i}^{\mathrm{r}}\left|g_{i}\right|^{2}} .
$$

Let us define the function $w(x) \triangleq\left(\sigma^{2}+x\right) /\left(\sigma^{2}+x\left|g_{i}\right|^{2}\right)$ for $x \geq 0$. The first derivative of $w(x)$ with respect to $x$ is calculated as

$$
\frac{\partial w(x)}{\partial x}=\frac{\sigma^{2}\left(1-\left|g_{i}\right|^{2}\right)}{\left(\sigma^{2}+x\left|g_{i}\right|^{2}\right)^{2}}>0 \text {, if and only if }\left|g_{i}\right|^{2}<1 \text {. }
$$

Therefore, we have

$$
w(x) \geq \min _{x \geq 0} w(x)=\min \left\{\frac{1}{\left|g_{i}\right|^{2}}, 1\right\} .
$$

Finally, by considering the $\bar{S}_{i}^{\mathrm{r}}$ in (4) as $x$, we obtain

$$
\gamma_{i} \geq \bar{\gamma}_{i}\left|h_{i}\right|^{2} \min \left\{\frac{1}{\left|g_{i}\right|^{2}}, 1\right\},
$$

which concludes the proof.

It can be shown that the bound proposed in Lemma 1 is tighter than the upper bound derived in [8]. The tightness will be further numerically evaluated in Section VI-C.

In this way, if the upper-bounded probability in (3) is smaller than $p_{0}$, the original inequality in (2) is always satisfied. Then, we further restrict the new outage probability requirement into the following two constraints,

$\operatorname{Pr}\left\{\sum_{i=1}^{E_{k^{\prime}}^{\text {all }}} \rho \log _{2}\left(1+\bar{\gamma}_{k^{\prime}}^{\mathrm{T}}\left|h_{i}\right|^{2} \min \left\{\frac{1}{\left|g_{i}\right|^{2}}, 1\right\}\right)<N_{k^{\prime}}\right\} \leq p_{\mathrm{o}}$

$\bar{\gamma}_{i} \geq \bar{\gamma}_{k^{\prime}}^{\mathrm{T}}, \quad \forall i=1,2, \ldots, E_{k^{\prime}}^{\mathrm{all}}$

\footnotetext{
${ }^{2}$ Note that $\bar{\gamma}_{i} \neq \mathbb{E}\left[\gamma_{i}\right]$, i.e., $\bar{\gamma}_{i}$ does not represent the average SINR
}

Constraints (8) and (9) mean that, for the $k^{\prime}$ th V-UE, by deriving $\bar{\gamma}_{k^{\prime}}^{\mathrm{T}}$ from (8) and forcing the actual $\bar{\gamma}_{i}$ on each used RB larger than $\bar{\gamma}_{k^{\prime}}^{\mathrm{T}}$, we can guarantee that (2) will be satisfied. Note that $\bar{\gamma}_{i}$ contains our decision variables that will be introduced later. From now on, with a slight abuse of terminology, we denote (9) as the SINR constraint.

For a given $\rho, N_{k^{\prime}}, p_{\mathrm{o}}$, and the probability density function (pdf) of $h_{i}$ as well as $g_{i}, \bar{\gamma}_{k^{\prime}}^{\mathrm{T}}$ and $E_{k^{\prime}}^{\text {all }}$ can be considered as functions of each other. Usually, the choice of $E_{k^{\prime}}^{\text {all }}$ depends on the traffic load of the network. In this paper, we assume a fixed $E_{k^{\prime}}^{\text {all }}$ and then derive $\bar{\gamma}_{k^{\prime}}^{\mathrm{T}}$ from $E_{k^{\prime}}^{\text {all }}$, e.g., by Monte Carlo (MC) simulation methods. The joint optimization of $E_{k^{\prime}}^{\text {all }}$ with other parameters in the proposed problem, which will be described later in Section IV, is left for future work.

Moreover, to meet the latency constraint, the $E_{k^{\prime}}^{\text {all }}$ RBs have to be allocated within the RB region $F \times L_{\text {tol }}$, as shown in Fig. 1, where $L_{\text {tol }}$ is the maximum tolerable latency of $\mathrm{V} 2 \mathrm{~V}$ communication in terms of the number of scheduling time units. Notice that in reality we have multiple V-UEs which may appear at different times. So it is hard to find a common two dimensional region to implement $\mathrm{RB}$ allocation for all the V-UEs. Therefore, we will reduce the two dimensional $\mathrm{RB}$ allocation problem into a sequence of one-dimensional problems, i.e., only over frequency. Correspondingly, the requirements on latency and reliability become

$$
\begin{aligned}
& E_{k^{\prime}}=\left\lceil E_{k^{\prime}}^{\mathrm{all}} / L_{\mathrm{tol}}\right\rceil \\
& \bar{\gamma}_{i} \geq \bar{\gamma}_{k^{\prime}}^{\mathrm{T}}, \quad \forall i=1,2, \ldots, E_{k^{\prime}},
\end{aligned}
$$

where $E_{k^{\prime}}$ is the number of RBs allocated to the $k^{\prime}$ th V-UE during each scheduling time unit, and we have $\sum_{k^{\prime}=1}^{K^{\prime}} E_{k^{\prime}} \leq$ $F$. The calculation of $E_{k^{\prime}}$ in (10) ensures that at least $E_{k^{\prime}}^{\text {all }}$ RBs will be allocated to the $k^{\prime}$ th V-UE within $L_{\text {tol }}$ time units.

In this way, we transformed the original $\mathrm{V} 2 \mathrm{~V}$ requirements on latency and reliability into constraints on $E_{k^{\prime}}$ and $\bar{\gamma}_{k^{\prime}}^{\mathrm{T}}$. To summarize, if the $k^{\prime}$ th V-UE is assigned $E_{k^{\prime}} \mathrm{RBs}$ during each time unit where the actual $\bar{\gamma}_{i}$ on the $i$ th used RB is larger than $\bar{\gamma}_{k^{\prime}}^{\mathrm{T}}$, then the original latency and reliability requirements will be satisfied for this V-UE.

\section{B. Requirements of $C$-UEs}

In contrast to $\mathrm{V} 2 \mathrm{~V}$ safety communications, for traditional cellular traffic, the latency requirement is less strict, and the system usually aims at maximizing the sum throughput under certain fairness considerations. Therefore, the maximization of the C-UEs' sum rate (as defined in Section IV) will be used as the objective of our optimization problem.

With regard to fairness, we assume proportional bandwidth fairness [20] among C-UEs, i.e., the number of RBs allocated to the $m^{\prime}$ th $\mathrm{C}$-UE, $E_{m^{\prime}}^{\prime}$, during one scheduling time unit is given for all $m^{\prime} \in \mathcal{M}^{\prime}$ and $\sum_{m^{\prime}=1}^{M^{\prime}} E_{m^{\prime}}^{\prime}=F$.

\section{Problem Formulation}

In this section, we detail the RRM problem formulation for D2D-based V2V communication, which considers the requirements of $\mathrm{V}$-UEs and $\mathrm{C}$-UEs at the same time. To summarize, our objective is to maximize the C-UEs' sum rate 
with fairness considerations, under the condition of satisfying V-UEs' requirements on latency and reliability, i.e., constraints (10) and (11).

To handle the allocation of multiple RBs for one UE, we introduce the concepts of sub-users and extended user sets. Firstly, we include one dummy V-UE, i.e., the $\left(K^{\prime}+1\right)$ th VUE, with the number of allocated RBs being $E_{K^{\prime}+1}=F-$ $\sum_{k^{\prime}=1}^{K^{\prime}} E_{k^{\prime}}$. Besides, to complete the dummy V-UE related information, we let $H_{K^{\prime}+1}=+\infty, G_{K^{\prime}+1}^{\prime}=0, \bar{\gamma}_{K^{\prime}+1}^{\mathrm{T}}=0$, and $G_{m^{\prime}\left(K^{\prime}+1\right)}=0$ for all $m^{\prime} \in \mathcal{M}^{\prime}$. Then, we divide the $k^{\prime}$ th V-UE into $E_{k^{\prime}}$ sub-V-UEs for all $k^{\prime} \in \tilde{\mathcal{K}} \triangleq\left\{1,2, \ldots, K^{\prime}+1\right\}$, and divide the $m^{\prime}$ th C-UE into $E_{m^{\prime}}^{\prime}$ sub-C-UEs for all $m^{\prime} \in$ $\mathcal{M}^{\prime}$, where each sub-user uses exactly one RB. Moreover, we define two extended user sets $\mathcal{K} \triangleq\{1,2, \ldots, F\}$ and $\mathcal{M} \triangleq$ $\{1,2, \ldots, F\}$ for sub-V-UEs and sub-C-UEs, respectively. In this way, we have $K=M=F$, where $K=|\mathcal{K}|$ and $M=$ $|\mathcal{M}|$. To relate the original user sets and the extended user sets, we define $\hat{k}: \mathcal{K} \rightarrow \tilde{\mathcal{K}}$ such that $k^{\prime}=\hat{k}(k)$ is the V-UE to which the sub-V-UE $k$ belongs. Similarly, the function $\hat{m}: \mathcal{M} \rightarrow \mathcal{M}^{\prime}$ is such that $m^{\prime}=\hat{m}(m)$ is the C-UE to which the sub-C-UE $m$ belongs. We further define $\mathcal{K}_{k^{\prime}} \triangleq\left\{k \mid k \in \mathcal{K}, \hat{k}(k)=k^{\prime}\right\}$ and $\mathcal{M}_{m^{\prime}} \triangleq\left\{m \mid m \in \mathcal{M}, \hat{m}(m)=m^{\prime}\right\}$ as the collection of sub-users for the $k^{\prime}$ th V-UE and $m^{\prime}$ th C-UE, respectively.

Based on the above definitions, the problem is mathematically formulated as maximizing the C-UEs' sum rate, i.e.,

$$
\max \sum_{m=1}^{M} \sum_{f=1}^{F} \log _{2}\left(1+\frac{S_{f m} H_{\hat{m}(m)}^{\prime}}{\sigma^{2}+\sum_{k=1}^{K} P_{f k} G_{\hat{k}(k)}^{\prime}}\right)
$$

subject to

$$
\begin{aligned}
& q_{f k} \in\{0,1\}, \quad l_{f m} \in\{0,1\}, \quad \forall f, k, m \\
& \sum_{f=1}^{F} \sum_{k \in \mathcal{K}_{k^{\prime}}} P_{f k} \leq P^{\max }, \quad \forall k^{\prime} \\
& \sum_{f=1}^{F} \sum_{m \in \mathcal{M}_{m^{\prime}}} S_{f m} \leq S^{\max }, \quad \forall m^{\prime} \\
& 0 \leq P_{f k} \leq P^{\max } q_{f k}, \quad \forall f, k \\
& 0 \leq S_{f m} \leq S^{\max _{f m}, \quad \forall f, m} \\
& \sum_{k=1}^{K} q_{f k}=1, \quad \sum_{m=1}^{M} l_{f m}=1, \quad \forall f \\
& \sum_{f=1}^{F} q_{f k}=1, \quad \sum_{f=1}^{F} l_{f m}=1, \quad \forall k, m \\
& \frac{P_{f k} H_{\hat{k}(k)}}{\sigma^{2}+\sum_{m=1}^{M} S_{f m} G_{\hat{m}(m) \hat{k}(k)}} \geq q_{f k} \bar{\gamma}_{\hat{k}(k)}^{\mathrm{T}}, \quad \forall f, k
\end{aligned}
$$

where $f \in \mathcal{F}, k \in \mathcal{K}, m \in \mathcal{M}, k^{\prime} \in \tilde{\mathcal{K}}$, and $m^{\prime} \in \mathcal{M}^{\prime}$. Moreover, $q_{f k}\left(l_{f m}\right)$ is a binary variable equal to 1 if the $k$ th sub-V-UE ( $m$ th sub-C-UE) is assigned to the $f$ th RB and 0 otherwise; $P_{f k}\left(S_{f m}\right)$ is the transmit power of the $k$ th sub-VUE ( $m$ th sub-C-UE) on the $f$ th RB. (12b) and (12c) represent the max transmit power constraints for each V-UE and CUE, respectively. Constraint (12d) (constraint (12e)) forces the transmit power of the $k$ th sub-V-UE (the $m$ th sub-C-UE) on the $f$ th RB to be 0 in case $q_{f k}=0\left(l_{f m}=0\right)$. (12f) guarantees the orthogonal RB allocation among V-UEs and among CUEs. (12g) ensures the number of RBs assigned to each subV-UE and each sub-C-UE is exactly one. Last but not least, (12h) enforces the SINR constraint for each sub-V-UE, where the LHS is interpreted as $\bar{\gamma}_{k}$.

In problem (12), the inputs are $F, M^{\prime}, K^{\prime}, E_{m^{\prime}}^{\prime}, E_{k^{\prime}}, \sigma^{2}$, $\bar{\gamma}_{k^{\prime}}^{\mathrm{T}}, P^{\max }, S^{\max }, H_{m^{\prime}}^{\prime}, G_{k^{\prime}}^{\prime}, H_{k^{\prime}}$, and $G_{m^{\prime} k^{\prime}}$. The outputs (also the optimization/decision variables) are $q_{f k}, l_{f m}, P_{f k}$, and $S_{f m}$ for all $f \in \mathcal{F}, k \in \mathcal{K}$, and $m \in \mathcal{M}$.

Unfortunately, the proposed problem (12) is NP-hard, which can be shown by reducing a partition problem into an instance of problem (12). As a result, there is no polynomial time algorithm to solve problem (12) optimally (unless $\mathrm{P}=\mathrm{NP}$ ), and thus heuristic solutions will be applied here.

\section{RRM SOLUTIONS}

In this section, we will first propose a novel RRM scheme called SOLEN in Section V-A to solve problem (12). In Section V-B, we will show how to extend the algorithm in [16] for its application to D2D-based V2V communication.

\section{A. The Proposed SOLEN Algorithm}

There are two stages in the Separate resOurce bLock and powEr allocatioN (SOLEN) algorithm. Firstly, by replacing the max sum power constraint for each UE with the max power constraint on each sub-user, the eNB allocates RBs to both V-UEs and C-UEs in an optimal and time efficient way by transforming the RB allocation problem into an maximum weight matching (MWM) problem for bipartite graphs. See [21] for general background on MWM. Secondly, based on the RB allocation results from the first stage, the eNB further optimally adjusts the transmit power for each V-UE and CUE when taking the max sum power constraint into account. This is realized via transforming the power allocation problem into a convex optimization problem and then solving it with a dual decomposition method [22] which can be efficiently computed. In this way, even though the proposed SOLEN method is heuristic by dividing the whole process into two stages, we can achieve the optimal solution for each stage, which to some extent promises good performance of the SOLEN algorithm, which is indeed confirmed by numerical results in Section VI-C.

In the following, we first derive the RB allocation scheme in Section V-A1, then present the power allocation algorithm in Section V-A2, and finally summarize the proposed SOLEN scheme in Section V-A3.

1) RB Allocation: Initially, the max sum power constraints (12b) and (12c) for each V-UE and C-UE are replaced with certain other constraints. In our previous work [8], we consider equal power allocation for each V-UE and C-UE on each of their used RBs in the RB allocation stage. In this paper, instead, we assume max power constraints for each V-UE and C-UE on each of their used RBs, i.e., for the $k^{\prime}$ th V-UE, the max power on each of its used RBs is $\check{P}_{k^{\prime}}^{\max } \triangleq P^{\max } / E_{k^{\prime}}$. 
Likewise, for the $m^{\prime}$ th C-UE, the max power on each of its used RBs is $\check{S}_{m^{\prime}}^{\max } \triangleq S^{\max } / E_{m^{\prime}}^{\prime}$. In this way, the constraints (12b) and (12c) will be replaced with $P_{f k} \leq \check{P}_{\hat{k}(k)}^{\max }$ and $S_{f m} \leq \check{S}_{\tilde{m}(m)}^{\max }$, respectively; and the new problem, which is similar to the formulation in (12) by including the updated constraints (12b) and (12c), is denoted as the RB allocation problem.

In the following, we will transform the RB allocation problem into another equivalent formulation ${ }^{3}$, and then present an algorithm to solve the equivalent problem optimally and efficiently. The basic track is as follows. Firstly, Theorem 1 is proposed to prove that the RB allocation problem can be transformed into the optimization problem (13) to get rid of the RB index $f$. Furthermore, we will propose Lemma 2 to show that problem (13) can be transformed into an MWM problem for bipartite graphs, and thus can be solved optimally by the Hungarian algorithm [21].

Theorem 1. The RB allocation problem can be transformed into the following equivalent optimization problem (13).

$$
\max \sum_{m=1}^{M} \sum_{k=1}^{K} x_{m k} \log _{2}\left(1+\frac{S_{m} H_{\hat{m}(m)}^{\prime}}{\sigma^{2}+P_{k} G_{\hat{k}(k)}^{\prime}}\right)
$$

subject to

$$
\begin{aligned}
& x_{m k} \in\{0,1\}, \quad \forall m \in \mathcal{M}, k \in \mathcal{K} \\
& \sum_{k=1}^{K} x_{m k}=1, \quad \forall m \in \mathcal{M}, \quad \sum_{m=1}^{M} x_{m k}=1, \quad \forall k \in \mathcal{K} \\
& S_{m} \leq \check{S}_{\hat{m}(m)}^{\max }, \quad \forall m \in \mathcal{M}, \quad P_{k} \leq \check{P}_{\hat{k}(k)}^{\max }, \forall k \in \mathcal{K} \\
& \frac{x_{m k} P_{k} H_{\hat{k}(k)}}{\sigma^{2}+S_{m} G_{\hat{m}(m) \hat{k}(k)}} \geq x_{m k} \bar{\gamma}_{\hat{k}(k)}^{\mathrm{T}}, \forall m \in \mathcal{M}, k \in \mathcal{K},
\end{aligned}
$$

where the optimization variables are $x_{m k}, S_{m}$, and $P_{k}$ for all $m \in \mathcal{M}$ and $k \in \mathcal{K}$; and the outputs are $x_{m k}$ for all $m \in \mathcal{M}$ and $k \in \mathcal{K}$.

Proof: See Appendix A.

In fact, problem (13) has its own meaning. Based on the definition of sub-users, the binary variable $x_{m k}$ is equal to 1 if the $m$ th sub-C-UE and the $k$ th sub-V-UE are sharing the same RB and is equal to 0 otherwise. Also, each sub-C-UE is required to share the same RB with exactly one sub-V-UE, and vice versa. Besides, $S_{m}$ and $P_{k}$ stand for the transmit power of the $m$ th sub-C-UE and the $k$ th sub-V-UE, respectively. Then, problem (13) is maximizing the sum rate of sub-C-UEs under the condition of satisfying the max power constraint on each RB for each sub-user, and the SINR constraint for each sub-VUE. Note that, even though $x_{m k}, S_{m}$, and $P_{k}$ are all optimization variables, the output of problem (13) only involves $x_{m k}$, while $S_{m}$ and $P_{k}$ serve as intermediate variables. Furthermore, as analyzed in Section II-B, the available channel information of all the involved links is the same in the whole considered

\footnotetext{
${ }^{3}$ As in [23], here we use the notion of equivalence of optimization problems in an informal way. We call two problems equivalent if from a solution of one, a solution of the other is readily found, and vice versa.
}

frequency range. Also, the SSF is assumed to be independent over different RBs. Hence, after pairing a sub-C-UEs with the corresponding sub-V-UEs, there is no difference which $\mathrm{RB}$ each pair is using as long as different pairs are using orthogonal RBs ${ }^{4}$.

In a further way, we will propose Lemma 2 to reformulate problem (13) into the pure integer program (14) which fits the MWM for bipartite graphs.

Lemma 2. Problem (13) is equivalent to

$$
\begin{aligned}
& \max \sum_{m=1}^{M} \sum_{k=1}^{K} x_{m k} \log _{2}(1+ \\
& \left.\frac{T_{m k}^{*} H_{\hat{m}(m)}^{\prime} H_{\hat{k}(k)}}{\sigma^{2} H_{\hat{k}(k)}+G_{\hat{k}(k)}^{\prime} \bar{\gamma}_{\hat{k}(k)}^{\mathrm{T}} \sigma^{2}+T_{m k}^{*} G_{\hat{k}(k)}^{\prime} \bar{\gamma}_{\hat{k}(k)}^{T} G_{\hat{m}(m) \hat{k}(k)}}\right),
\end{aligned}
$$

subject to (13a) and (13b), where

$T_{m k}^{*}=\min \left\{\check{S}_{\hat{m}(m)}^{\max }, \frac{\check{P}_{\hat{k}(k)}^{\max } H_{\hat{k}(k)}-\bar{\gamma}_{\hat{k}(k)}^{\mathrm{T}} \sigma^{2}}{\bar{\gamma}_{\hat{k}(k)}^{T} G_{\hat{m}(m) \hat{k}(k)}}\right\}, \forall m \in \mathcal{M}, k \in \mathcal{K}$.

Proof: Since the objective function (13) is nonincreasing in terms of $\left\{P_{k}\right\}_{k=1}^{K}$, the optimal $\check{P}_{k}^{*}$ must be achieved at

$$
\check{P}_{k}^{*}=\frac{1}{H_{\hat{k}(k)}} \sum_{m=1}^{M} x_{m k}\left(\bar{\gamma}_{\hat{k}(k)}^{\mathrm{T}} \sigma^{2}+S_{m} \bar{\gamma}_{\hat{k}(k)}^{\mathrm{T}} G_{\hat{m}(m) \hat{k}(k)}\right), \forall k \in \mathcal{K}
$$

which is obtained from (13d). Then, by substituting $\check{P}_{k}^{*}$ into (13), we can eliminate $\left\{P_{k}\right\}_{k=1}^{K}$ and transform the objective function in (13) into

$$
\begin{aligned}
& \sum_{m=1}^{M} \sum_{k=1}^{K} x_{m k} \log _{2}[1+ \\
& \left.\frac{S_{m} H_{\hat{m}(m)}^{\prime} H_{\hat{k}(k)}}{\sigma^{2} H_{\hat{k}(k)}+G_{\hat{k}(k)}^{\prime} \sum_{m=1}^{M} x_{m k}\left(\bar{\gamma}_{\hat{k}(k)}^{\mathrm{T}} \sigma^{2}+S_{m} \bar{\gamma}_{\hat{k}(k)}^{\mathrm{T}} G_{\hat{m}(m) \hat{k}(k)}\right)}\right] \\
& =\sum_{m=1}^{M} \sum_{k=1}^{K} x_{m k} \log _{2}(1+ \\
& \left.\frac{T_{m k} H_{\hat{m}(m)}^{\prime} H_{\hat{k}(k)}}{\sigma^{2} H_{\hat{k}(k)}+G_{\hat{k}(k)}^{\prime} \bar{\gamma}_{\hat{k}(k)}^{\mathrm{T}} \sigma^{2}+T_{m k} G_{\hat{k}(k)}^{\prime} \bar{\gamma}_{\hat{k}(k)}^{\mathrm{T}} G_{\hat{m}(m) \hat{k}(k)}}\right),
\end{aligned}
$$

where $T_{m k} \triangleq x_{m k} S_{m}$.

It can be shown that (18) is nondecreasing in terms of $T_{m k} \geq 0$. Hence, by taking into account the constraint (13c), the maximum of (18) must be reached at the optimal $T_{m k}^{*}$ in (15), which concludes the proof.

Now, due to the properties of the MWM for bipartite graphs, the Hungarian algorithm [21] is an efficient way to solve problem (14) and derive the optimal solution $x_{m k}^{*}$ within

\footnotetext{
${ }^{4}$ The instantaneous rate, in fact, depends on which RB to use due to SSF effects. However, this SSF knowledge is not available at the eNB, and thus the eNB cannot include the SSF information when optimizing the allocation.
} 
polynomial time, where the number of operations is upperbounded by $O\left(F^{3}\right)[21]$.

2) Power Allocation: The second stage of the proposed SOLEN algorithm is power allocation. According to $x_{m k}^{*}$ obtained by solving problem (14), we define $\bar{k}^{*}: \mathcal{M} \rightarrow \mathcal{K}$ such that $\bar{k}^{*}(m)$ is the sub-V-UE which is sharing the same RB with $m$ th sub-C-UE. Similarly, the function $\bar{m}^{*}: \mathcal{K} \rightarrow \mathcal{M}$ is such that $\bar{m}^{*}(k)$ is the sub-C-UE which is sharing the same $\mathrm{RB}$ with $k$ th sub-V-UE. In this way, the power allocation problem can be formulated as

$$
\max \sum_{m=1}^{M} \log _{2}\left(1+\frac{S_{m} H_{\hat{m}(m)}^{\prime}}{\sigma^{2}+P_{\bar{k}^{*}(m)} G_{\hat{k}\left(\bar{k}^{*}(m)\right)}^{\prime}}\right)
$$

subject to

$$
\begin{aligned}
& S_{m} \geq 0, \quad P_{k} \geq 0, \quad \forall m \in \mathcal{M}, k \in \mathcal{K} \\
& \sum_{m \in \mathcal{M}_{m^{\prime}}} S_{m} \leq S^{\max }, \quad \forall m^{\prime} \in \mathcal{M}^{\prime} \\
& \sum_{k \in \mathcal{K}_{k^{\prime}}} P_{k} \leq P^{\max }, \quad \forall k^{\prime} \in \mathcal{K}^{\prime} \\
& \frac{P_{k} H_{\hat{k}(k)}}{\sigma^{2}+S_{\bar{m}^{*}(k)} G_{\hat{m}\left(\bar{m}^{*}(k)\right) \hat{k}(k)}} \geq \bar{\gamma}_{\hat{k}(k)}^{\mathrm{T}}, \quad \forall k \in \mathcal{K}
\end{aligned}
$$

where the optimization variables are $S_{m}$ and $P_{k}$ for all $m \in \mathcal{M}$ and $k \in \mathcal{K}$. Note that the original max sum power constraints (12d) and (12e) are included in the power allocation problem. Since the objective function in (19) is not concave with respect to $\left\{P_{k}\right\}_{k=1}^{K}$, this problem is a non-convex optimization problem. Nevertheless, we propose Lemma 3 to transform the power allocation problem (19) into the convex optimization problem (20).

Lemma 3. Given the RB allocation results, the power allocation problem (19) can be transformed into the convex optimization problem (20).

Proof: Similar to the proof of Lemma 2, the optimal $P_{k}^{*}$ is achieved by satisfying the equality point in (19d). Then, substitute $P_{k}^{*}$ into the power allocation problem (19) and set $k=\bar{k}^{*}(m)$, we can eliminate $\left\{P_{k}\right\}_{k=1}^{K}$ and transform (19) into (20) (on bottom of this page), subject to

$$
\begin{aligned}
& S_{m} \geq 0, \quad \forall m \in \mathcal{M}, \\
& \sum_{m \in \mathcal{M}_{m^{\prime}}} S_{m} \leq S^{\max }, \quad \forall m^{\prime} \in \mathcal{M}^{\prime} \\
& \sum_{\substack{m: \bar{k}^{*}(m) \in \mathcal{K}_{k^{\prime}} \\
\leq}} \frac{\bar{\gamma}_{\hat{k}\left(\bar{k}^{*}(m)\right)}^{\mathrm{T}} \sigma^{2}}{H_{\hat{k}\left(\bar{k}^{*}(m)\right)}}+S_{m} \frac{\bar{\gamma}_{\hat{k}\left(\bar{k}^{*}(m)\right)}^{\mathrm{T}} G_{\hat{m}(m) \hat{k}\left(\bar{k}^{*}(m)\right)}}{H_{\hat{k}\left(\bar{k}^{*}(m)\right)}} \quad \forall k^{\prime} \in \mathcal{K}^{\prime}
\end{aligned}
$$

where the optimization variables are $S_{m}$ for all $m \in \mathcal{M}$.
Via verifying the positive semidefinite property of the corresponding Hessian matrix, it can be proved that the objective function in (20) is convex with respect to $\left\{S_{m}\right\}_{m=1}^{M}$. It can also be shown that the feasible set decided by constraints (20a)(20c) is a convex set. Therefore, the transformed problem (20) is a convex optimization problem.

Even though problem (20) is convex and we can adopt some well known algorithms, e.g., interior point method or Newton's method, to solve it optimally, it may require high complexity especially for large size problems. Therefore, we will utilize the dual decomposition method [22] to separate problem (20) into subproblems that can be solved in parallel. For a convex optimization problem with satisfied Slater's condition [22], the dual decomposition method can guarantee the optimal solution as the strong duality holds for this case.

Detailed derivations of the proposed power allocation scheme are given in Appendix B. For readers' convenience, the processes are presented in Algorithm 1.

$\overline{\text { Algorithm } 1 \text { Proposed power allocation algorithm to solve }}$

Input: $\bar{\gamma}_{k^{\prime}}^{\mathrm{T}}, H_{m^{\prime}}^{\prime}, G_{k^{\prime}}^{\prime}, H_{k^{\prime}}$, and $G_{m^{\prime} k^{\prime}}$ for all $m^{\prime} \in \mathcal{M}^{\prime}$ and $k^{\prime} \in \mathcal{K}^{\prime}, \sigma^{2}, S^{\max }$, and $P^{\max }$

Output: $S_{m}^{*}$ for all $m \in \mathcal{M}$

Initialization: (i) define $z, a_{m}, b_{m}, c_{m}, v_{k^{\prime} m}$, and $r_{k^{\prime} m}$ for all $m \in \mathcal{M}$ and $k^{\prime} \in \mathcal{K}^{\prime}$ based on (28)-(33) in Appendix B;

(ii) set $t=0$ and $\boldsymbol{\lambda}^{(0)}$ equal to some nonnegative vector.

1. Solve subproblems (38) (in parallel) for given $\boldsymbol{\lambda}^{(t)}$. To solve the $m^{\prime}$ th subproblem, calculate $\tilde{z}_{m}^{*}(\boldsymbol{\lambda})$ from (41),

if $\sum_{m \in \mathcal{M}_{m},} \tilde{z}_{m}^{*} \leq S^{\max }$ then $z_{m}^{*}(\boldsymbol{\lambda})=\tilde{z}_{m}^{*}(\boldsymbol{\lambda})$

else

a) derive $\mu_{m^{\prime}}^{*}$ from (56),

b) calculate $z_{m}^{*}(\boldsymbol{\lambda})$ from (55)

end if

2. Update $\boldsymbol{\lambda}$ by $\boldsymbol{\lambda}^{(t+1)}=\left[\boldsymbol{\lambda}^{(t)}-\alpha^{(t)} \sum_{m^{\prime}=1}^{M^{\prime}} \boldsymbol{\eta}_{m^{\prime}}\left(\boldsymbol{\lambda}^{(t)}\right)\right]$, where $\alpha^{(t)}$ is an appropriate step size, and $\boldsymbol{\eta}_{m^{\prime}}\left(\boldsymbol{\lambda}^{(t)}\right)$ is obtained from (40).

3. $t \leftarrow t+1$ and go to step 1 (until satisfying termination criterion).

4. Obtain $S_{m}^{*}$ from $z^{*}$.

Remark 1. In fact, there is a hard upper limit on the acceptable number of $V$-UEs for different $E_{k^{\prime}}$, since $V$-UEs can only use orthogonal RBs among each other in this work. For instance, when $F=100$, the upper limits on $K^{\prime}$ are 50 and 20 for $E_{k^{\prime}}=2$ and $E_{k^{\prime}}=5$, respectively. Due to the stringent requirements of $V 2 \mathrm{~V}$ communications, it is

$$
\min -\sum_{m=1}^{M} \log _{2}\left(1+\frac{S_{m} H_{\hat{m}(m)}^{\prime} H_{\hat{k}^{(}\left(\bar{k}^{*}(m)\right)}}{\sigma^{2} H_{\hat{k}\left(\bar{k}^{*}(m)\right)}+G_{\hat{k}\left(\bar{k}^{*}(m)\right)}^{\prime} \bar{\gamma}_{\hat{k}\left(\bar{k}^{*}(m)\right)}^{\mathrm{T}} \sigma^{2}+S_{m} G_{\hat{k}\left(\bar{k}^{*}(m)\right)}^{\prime} \bar{\gamma}_{\hat{k}\left(\bar{k}^{*}(m)\right)}^{\mathrm{T}} G_{\hat{m}(m) \hat{k}\left(\bar{k}^{*}(m)\right)}}\right)
$$


of paramount importance to notify the V-UE if it can be serviced or not, which depends on the feasibility of problem (12). Obviously, problem (12) is infeasible if $\sum_{k^{\prime}=1}^{K^{\prime}} E_{k^{\prime}}>F$. Hence, as in [24], we can use an Availability Indicator to include the information of the feasibility ${ }^{5}$.

3) Summary of the SOLEN scheme: To summarize, the procedures of the proposed SOLEN algorithm for solving problem (12) are illustrated in Algorithm 2.

\section{B. Application of [16] to D2D-based V2V}

As stated in Section I-C, the proposed mathematical model for V-UE requirements opens the feasibility of applying the existing D2D techniques to $\mathrm{V} 2 \mathrm{~V}$ scenarios. Now we will extend the algorithm in [16] for its application in D2D-based V2V communication, which is referred to V2V-[16]. Firstly, we involve the constraints on the number of RBs and the SINR target value for V-UEs, which are derived in Section III-A, into its problem formulation. Then, the concepts of sub-C-UE and sub-V-UE are introduced to allow the allocation of multiple RBs for one UE. Correspondingly, the max power constraints become $\check{S}_{m^{\prime}}^{\max }$ and $\check{P}_{k^{\prime}}^{\max }$ for each sub-C-UE and each sub-VUE, respectively. Furthermore, we change the objective in the second and third steps of the scheme from maximizing the sum rate of both $\mathrm{C}$-UEs and V-UEs into maximizing the sum rate of $\mathrm{C}$-UEs, and also revise the derivations of the algorithm accordingly.

\section{PERFormance Evaluation}

\section{A. Scenarios and Parameters}

We assume a single cell outdoor system with a carrier frequency of $800 \mathrm{MHz}$ and that each RB has a bandwidth of $180 \mathrm{kHz}$ for uplink communication. In particular, we consider test case 2 [25] defined by METIS, which describes an urban environmental model similar to the Manhattan grid layout. In this topology, the entire region is a $444 \mathrm{~m} \times 444 \mathrm{~m}$ square and the size of each building is $120 \mathrm{~m} \times 120 \mathrm{~m}$.

The used channel models are specified by [25], which describes the large scale modeling for different propagations scenarios (PSs). Specifically, we refer to PS\#3 in [25] for the links connected to the eNB (i.e., $H_{m^{\prime}}^{\prime}$ and $G_{k^{\prime}}^{\prime}$ ); and PS\#9 in [25] for the links between UEs (i.e., $H_{k^{\prime}}$ and $G_{m^{\prime} k^{\prime}}$ ).

Simulation parameters are summarized as follows: $\rho=84$, $P^{\max }=S^{\max }=24 \mathrm{dBm}$. Besides, the antenna height is 26 $\mathrm{m}$ at the eNB and $1.5 \mathrm{~m}$ at each UE. The intended broadcast range of each vehicle is $18 \mathrm{~m}$. Also, the noise floor is -117 $\mathrm{dBm}$ at the eNB and each V-UE. The SSF of the channels is assumed to be Rayleigh distributed with unit power gain.

\footnotetext{
${ }^{5}$ How to best resolve an infeasibility situation, e.g., by offering the service to only a subset of V-UEs, is application-dependent and outside the scope of this paper.

${ }^{6}$ As analyzed in Section V-A1, after pairing the sub-C-UEs with the corresponding sub-V-UEs, there is no difference in which RB each pair is using as long as different pairs are using orthogonal RBs. This is because the available channel information of all the involved links merely includes the slow fading effects, which are roughly the same in the whole considered frequency range. Also, the SSF is assumed to be independent over different RBs.
}

Algorithm 2 Procedures of the proposed SOLEN algorithm to solve problem (12)

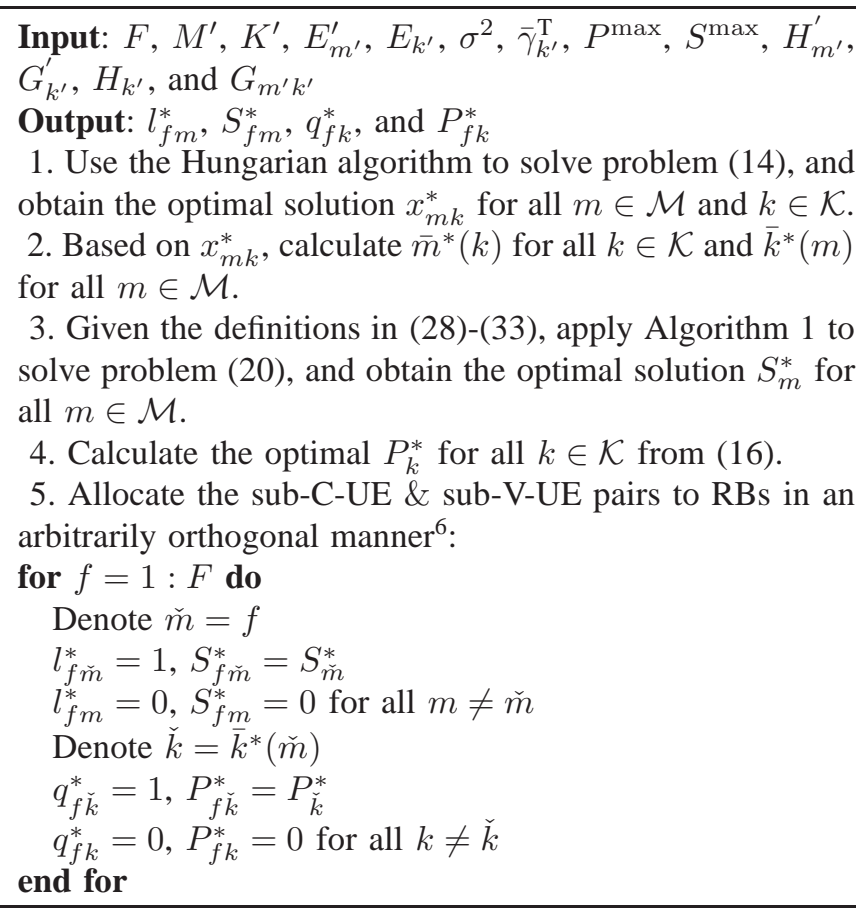

Finally, one scheduling time unit (i.e., the time period of one $\mathrm{RB}$ ) is $0.5 \mathrm{~ms}$ and the time scale of RRM is $100 \mathrm{~ms}$.

\section{B. Performance Metrics and Baseline Methods}

We base our evaluation on four metrics:

- C-UEs' sum rate when SSF is disregarded, i.e., the value of the objective in (12);

- transmit power per V-UE and per C-UE;

- cumulative distribution function (CDF) of C-UEs' sum rate;

- CDF of one V-UE's transmitted bits within $5 \mathrm{~ms}$, i.e., the LHS of the inner inequality in (1).

The last two metrics are evaluated when considering SSF in simulations.

Moreover, we compare the SOLEN and V2V-[16] with the following baseline methods.

1) Modified-[12]. In [12], the eNB selects the C-UE with highest desired channel gain to share its $\mathrm{RB}$ with the V-UE which suffers the lowest interference from this C-UE. The method is executed with the max power. To fit the scheme into our framework, we first use the concepts of sub-C-UE and sub-V-UE instead of C-UE and V-UE. Besides, to meet the SINR constraint for each sub-V-UE, we simply decrease the transmit power of the corresponding sub-C-UEs until the SINR constraint is just satisfied.

2) Modified-[16]. In [16], a three-step scheme is derived to maximize the sum rate of both $\mathrm{C}$-UEs and V-UEs. Here, we replace $\mathrm{C}$-UEs and $\mathrm{V}$-UEs with sub-C-UEs and sub-V-UEs, respectively.

3) SRBP in [8]. In [8], it is firstly derived an upper bound on the outage probability $p_{k^{\prime}}^{\text {out }}$ in (1) using only the slowly 


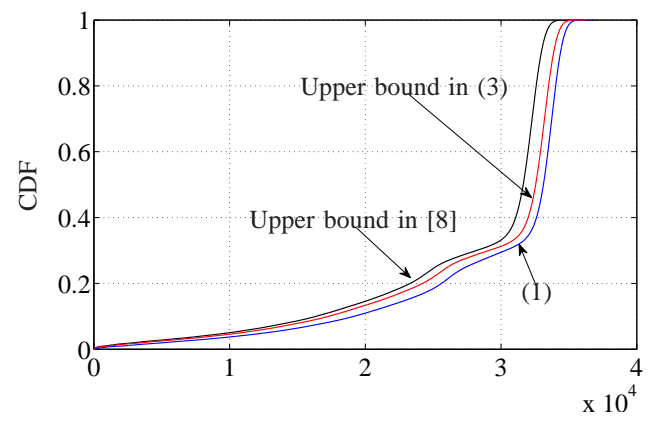

Figure 3. CDF of upper bounds.

varying channel information. Moreover, similar to SOLEN, it is also proposed a two-stage SRBP scheme which separates RB allocation and power control. However, in its RB allocation stage, power is equally allocated for each V-UE and C-UE on each of their used RBs.

4) Optimal solution to problem (12), which is achieved by firstly conducting the exhaustive search over all the RB allocation possibilities, and then implementing the optimal power allocation for each RB allocation result. Due to its exponentially increased complexity, we only simulate the optimal solution for $F=4$.

\section{Simulation Results}

Based on the requirements specified by METIS [1], we have $N_{k^{\prime}}=12800$ bits, $p_{\mathrm{o}}=10^{-5}$ (i.e., a transmission reliability of $99.999 \%$ ), and $L_{\text {tol }}=10$ (i.e., a latency requirement of $5 \mathrm{~ms}$ ). As analyzed in Section III-A, the relationship between $E_{k^{\prime}}^{\text {all }}$ and $\bar{\gamma}_{k^{\prime}}^{\mathrm{T}}$ can be derived from (8) through a MC method. Then $E_{k^{\prime}}$ can be calculated via (10). In this way, some possible values of $\left\{E_{k^{\prime}}, \bar{\gamma}_{k^{\prime}}^{\mathrm{T}}[\mathrm{dB}]\right\}$ are $\{2,32.6\},\{3,23.2\},\{4,18.2\},\{5,14.9\}$, $\{6,12.5\},\{7,10.8\},\{8,9.3\},\{8,9.3\}$, and $\{10,7.2\}$.

Recall that the constraint in (10) is obtained on the basis of Lemma 1. Therefore, we will first evaluate the tightness of the upper bound given in Lemma 1. To do so, we compare the CDFs of the random variables $\sum_{i=1}^{E_{k \prime}^{\text {all }}} \rho \log _{2}\left(1+\gamma_{i}\right)$ in (1), which corresponds to the actual outage probability, $\sum_{i=1}^{E_{k^{\prime}}^{\text {all }}} \rho \log _{2}\left(1+\bar{\gamma}_{i}\left|h_{i}\right|^{2} \min \left\{\frac{1}{\left.g_{i}\right|^{2}}, 1\right\}\right)$ given by the upper bound in (3), and the upper bound proposed in [8]. The numerical result shown in Fig. 3 demonstrates the validity of the bound derived by Lemma 1 and its improvement compared to the existing bound presented in [8].

Fig. 4 compares C-UEs' sum rates of different schemes when $F=4$, which is plotted to show the performance gap with the optimal solution. The numbers in the labels represent the achieved rates when the SSF is not taken into account. In other words, the rate when the utilized channel knowledge in the four RRM methods matches the actual channel in the simulations. Besides, the CDF curves show C-UEs' sum rates when the SSF is also involved in simulated channels. It can be seen that these long-term RRM schemes do not incur big difference on the average performance when being applied to realistic channels with SSF effects. Regarding the evaluation of different methods, the performances of SOLEN

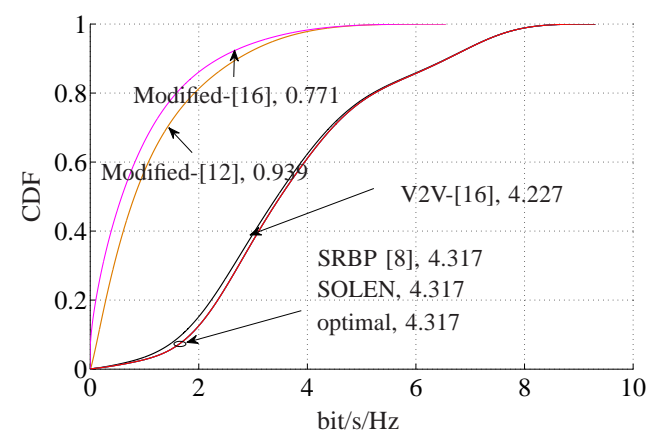

Figure 4. Sum rate of C-UEs. $F=4, M^{\prime}=1, E_{m^{\prime}}^{\prime}=4, K^{\prime}=2$, and $E_{k^{\prime}}=2$.

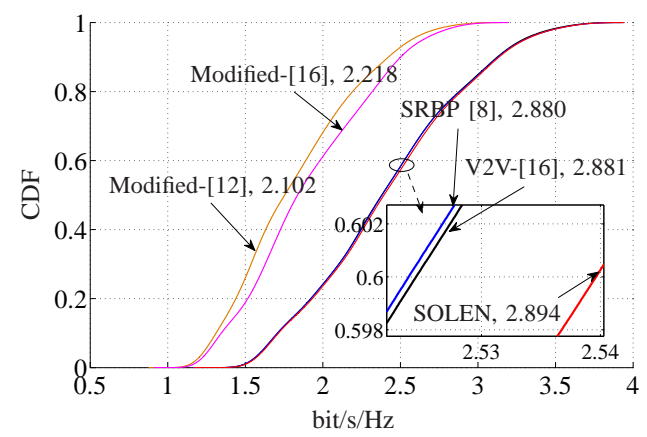

(a)

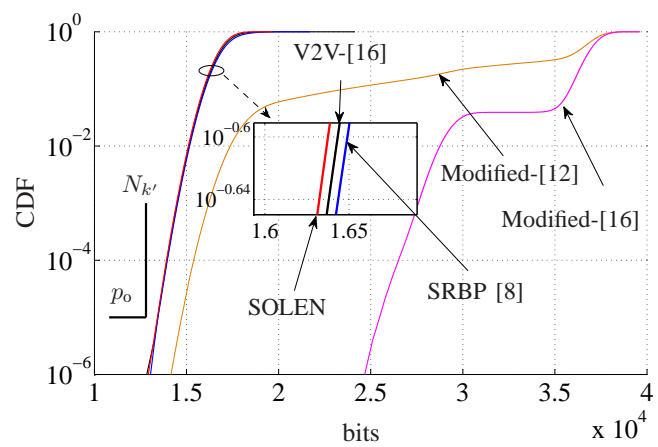

(b)

Figure 5. $F=100, M^{\prime}=5, E_{m^{\prime}}^{\prime}=20, K^{\prime}=10$, and $E_{k^{\prime}}=2$. (a) Sum rate of C-UEs. (b) CDF of transmitted bits within $5 \mathrm{~ms}$ for each V-UE.

and SRBP achieve the optimal solution in this setup. V2V[16] exhibits slight degradation. On the other hand, Modified[12] and Modified-[16] yield significantly worse sum rates, which illustrates the ineffectiveness of directly applying D2D schemes to V2V communication.

Now consider more realistic scenarios with $F=100$. Here we define the traffic load as the number of V-UEs. For a low load situation, i.e., $K^{\prime}=10$, Fig. 5(a) and Fig. 5(b) illustrate the performances of C-UEs and V-UEs, respectively.

In Fig. 5(a), the CDFs of C-UEs' sum rates are evaluated. By comparing the numbers in the labels and the CDF curves, it is again demonstrated that long-term RRM schemes yield effective results even when SSF effects are present. Besides, compared to V2V-[16], SRBP and SOLEN, Modified- [12] and Modified-[16] have obviously degraded performances due to the inappropriateness of their models for D2D-based V2V 


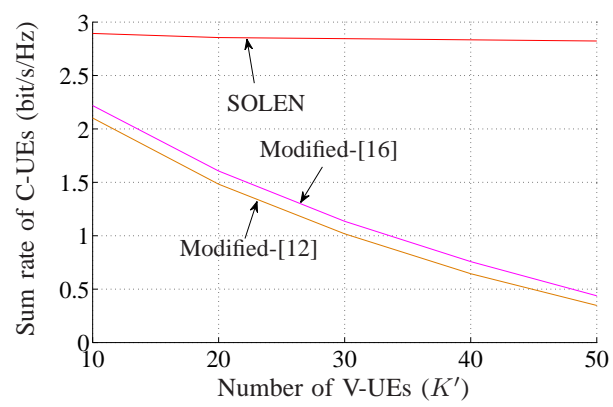

(a)

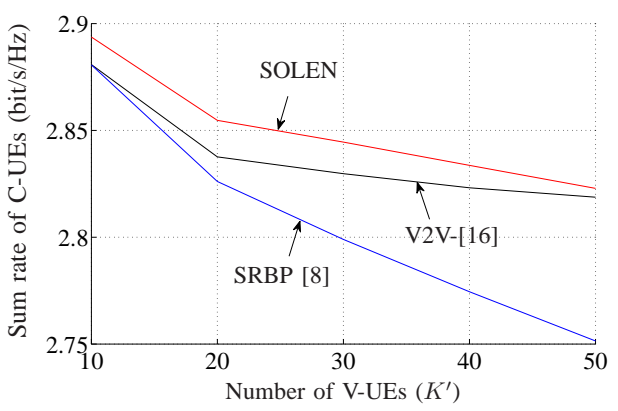

(b)

Figure 6. Sum rate of C-UEs versus number of V-UEs. $F=100, M^{\prime}=5$, $E_{m^{\prime}}^{\prime}=20$, and $E_{k^{\prime}}=2$. (a) Sum rate of C-UEs. (b) Zoom in.

communication. Moreover, SOLEN reveals slight superiority over SRBP and V2V-[16].

Fig. 5(b) shows the CDFs of the transmitted bits within 5 $\mathrm{ms}$ for one V-UE. It can be seen that the outage probability constraint which represents the QoS requirements on V-UEs is fulfilled for all the five schemes. We stress the fact that there is no need to exceed the requirements for V-UEs. Indeed, the fact that Modified-[12] and Modified-[16] do this to a higher degree than V2V-[16], SRBP, and SOLEN explains why their C-UE sum rates are worse in Fig. 5(a).

Next, C-UEs' sum rates are plotted in Fig. 6 with respect to different loads of vehicular network, i.e., different values of $K^{\prime}$. As revealed in Fig. 6(a), compared to the algorithms proposed for traditional D2D networks (i.e., Modified-[12] and Modified-[16]), the proposed SOLEN shows significant performance improvement and better robustness to network loads. This result again demonstrates the necessity of careful consideration when applying D2D network to vehicular communication. Furthermore, the three algorithms that are designed for D2D-based V2V communication are evaluated in Fig. 6(b), where SOLEN exhibits slight superiority as well. Besides, even though the best-performing SOLEN introduces higher computational complexity than V2V-[16] and SRBP due to the power allocation in the second stage, the power allocation problem is decomposed into subproblems that can be then solved in parallel. Furthermore, when $K^{\prime}$ is varied from 10 to 50, the satisfaction of V-UE's requirements will not be affected. Particularly, the CDF curves of the transmitted bits within $5 \mathrm{~ms}$ for each V-UE are similar with the ones shown in Fig. 5(b), and thus not included due to space limitation. The reason is that we consider the QoS requirements of V-UEs as

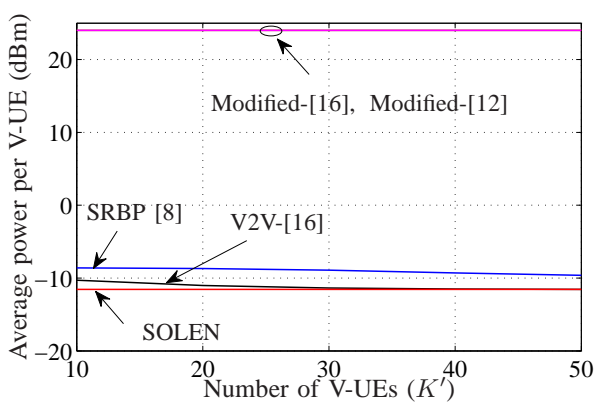

(a)

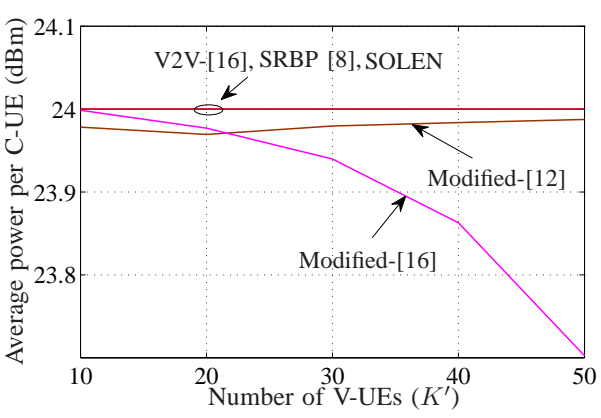

(b)

Figure 7. Average transmit power per UE versus number of V-UEs. $F=100$ $M^{\prime}=5, E_{m^{\prime}}^{\prime}=20$, and $E_{k^{\prime}}=2$. (a) Average transmit power per V-UE. (b) Average transmit power per C-UE.

hard constraints in the optimization problem (12) and render them higher priority over C-UE's sum rate.

Fig. 7(a) and Fig. 7(b) show the average transmit power in terms of $K^{\prime}$ per V-UE and per C-UE, respectively. First, from Fig. 7(a), it can be seen that V-UE's transmit power of Modified-[16] and Modified-[12] reaches the maximum, i.e., $24 \mathrm{dBm}$, for all $10 \leq K^{\prime} \leq 50$. Whereas, V-UE's transmit power of RRM schemes V2V-[16], SRBP [8], and SOLEN is much less than $24 \mathrm{dBm}$, where the proposed SOLEN algorithm yields the lowest power. Since the five schemes have the same QoS requirements of V-UEs, the lower transmit power per V-UE implies the better power efficiency. Besides, while the transmit power of V2V-[16] and SRBP [8] varies slightly with respect to different values of $K^{\prime}$, the power of SOLEN is almost a constant over the increased $K^{\prime}$ which illustrates its robustness to network loads. Second, when it comes to the average transmit power of C-UEs, as revealed in Fig. 7(b), V2V-[16], SRBP [8], and SOLEN achieve their max power, i.e., $24 \mathrm{dBm}$. This means that the sum rates of C-UEs of these three algorithms have been maximized as much as possible. However, Modified-[16] and Modified- [12] exhibit slightly less transmit power. This is because the C-UEs in these two schemes have to sacrifice the transmit power to guarantee the requirements of V-UEs, which again reveals the ineffectiveness of the naive extension when applying D2D methods to V2V communications.

Additionally, the CDF of V-UE's transmit power is shown by Fig. 8 for $K^{\prime}=30$. As revealed in Fig. 8, the transmit power per V-UE for RRM schemes Modified-[16] and Modified-[12] is constantly $24 \mathrm{dBm}$, and thus their respective 


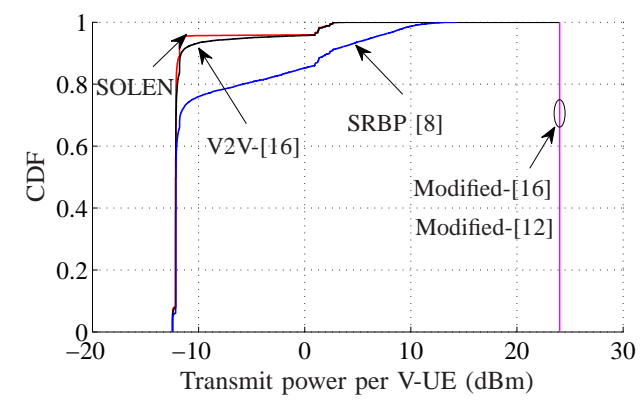

Figure 8. CDF of transmit power per V-UE versus number of V-UEs. $F=$ $100, M^{\prime}=5, E_{m^{\prime}}^{\prime}=20, K^{\prime}=30$, and $E_{k^{\prime}}=2$.

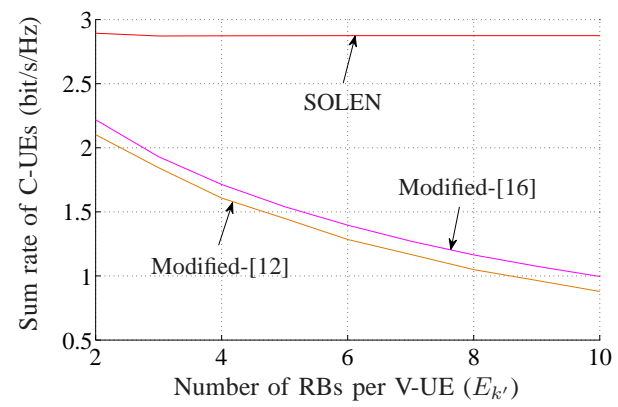

(a)

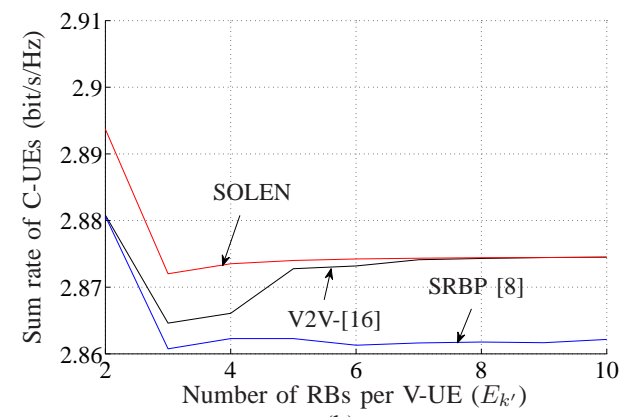

(b)

Figure 9. Sum rate of C-UEs versus number of RBs per V-UE. $F=100$, $M^{\prime}=5, E_{m^{\prime}}^{\prime}=20$, and $K^{\prime}=10$. (a) Sum rate of C-UEs. (b) Zoom in.

CDF is a step function with a 'step' at $24 \mathrm{dBm}$. Also, as illustrated by the CDF curves, the proposed SOLEN exhibits the best power efficiency. In fact, we have also evaluated the CDF of V-UE's transmit power for different $K^{\prime}$, i.e., $10 \leq K^{\prime} \leq 50$, and obtained almost identical simulation results with the case $K^{\prime}=30$. Hence. we will only include Fig. 8 here as an example.

Fig. 9 shows the impact of $E_{k^{\prime}}$ on the performances of RRM schemes. Recall that different $E_{k^{\prime}}$ gives different $\bar{\gamma}_{k^{\prime}}^{\mathrm{T}}$. Generally, increasing $E_{k^{\prime}}$ causes less transmit power of the V-UE on each of its used RBs due to the lowered $\bar{\gamma}_{k^{\prime}}^{\mathrm{T}}$, which then leads to the increased rate on that $\mathrm{RB}$ of the corresponding C-UE. On the other hand, increasing $E_{k^{\prime}}$ implies that more C-UEs' RBs suffer from interference caused by VUEs. Therefore, it is not obvious that what value of $E_{k^{\prime}}$ can bring the best performance of C-UEs' sum rate, which may vary based on different system parameters. Nevertheless, it is revealed in Fig. 9(a) that SOLEN dominates Modified-[12] and Modified-[16] under various $E_{k^{\prime}}$ possibilities. Moreover, as illustrated in Fig. 9(b), compared to V2V-[16], SRBP and SOLEN are more robust to the change of $E_{k^{\prime}}$. Last but not least, SOLEN outperforms all the other schemes and shows the most promising results.

\section{CONCLUSiOns AND Future WORK}

Due to the similarity between the QoS requirements of $\mathrm{V} 2 \mathrm{~V}$ application and the benefits of D2D communication, the direct D2D link is a promising enabler for $\mathrm{V} 2 \mathrm{~V}$ communication as long as the RRM is conducted in a careful way. In this paper, we first present a method to transform the actual latency and reliability requirements of $\mathrm{V} 2 \mathrm{~V}$ communication into optimization constraints that can be computed from only slowly varying CSI. This transformation allows us to apply certain existing D2D schemes to V2V communication, which, however, need to be revised to cater for V-UEs' specific requirements. Moreover, we formulate a problem to optimize the performance of both V-UEs and C-UEs, and propose the SOLEN algorithm to solve this problem. By doing so, C-UEs' sum rate can be maximized under the condition of satisfying V-UEs' constraints. Numerical results confirm that careful RRM design is necessary when applying D2D network to V2V communication. Moreover, the proposed SOLEN scheme shows promising performance.

The current work assumes the orthogonal RB allocation among V-UEs, i.e., the constraint in (12f). In the future, we will relax this restriction by allowing multiple V-UEs to share a common RB. By doing so, the number of supported V-UEs will improve; however, the possible non-orthogonality of $\mathrm{V}$ UEs will likely require a more complex RRM design.

\section{APPENDIX A PROOF OF THEOREM 1}

The proof starts by defining new variables $x_{m k f} \triangleq q_{f k} l_{f m}$ for all $f \in \mathcal{F}, k \in \mathcal{K}$ and $m \in \mathcal{M}$. By this definition, we can obtain the following constraints.

$$
\begin{aligned}
& x_{m k f} \in\{0,1\}, \quad \forall f \in \mathcal{F}, m \in \mathcal{M}, k \in \mathcal{K}, \\
& \sum_{f=1}^{F} x_{m k f} \in\{0,1\}, \quad \forall m \in \mathcal{M}, k \in \mathcal{K}, \\
& \sum_{m=1}^{M} \sum_{k=1}^{K} x_{m k f}=1, \quad \forall f \in \mathcal{F} .
\end{aligned}
$$

To prove the equivalent transformation from the RB allocation problem to problem (13), we need to take both objective function and constraints into account.

Let us first investigate the objective function in the $\mathrm{RB}$ allocation problem in (12), which is equal to

$$
\begin{aligned}
& \sum_{m=1}^{M} \sum_{f=1}^{F} l_{f m} \log _{2}\left(1+\frac{S_{f m} H_{\hat{m}(m)}^{\prime}}{\sigma^{2}+\sum_{k=1}^{K} q_{f k} P_{f k} G_{\hat{k}(k)}^{\prime}}\right) \\
& =\sum_{m=1}^{M} \sum_{f=1}^{F} \sum_{k=1}^{K} q_{f k} l_{f m} \log _{2}\left(1+\frac{S_{f m} H_{\hat{m}(m)}^{\prime}}{\sigma^{2}+\sum_{k=1}^{K} q_{f k} P_{f k} G_{\hat{k}(k)}^{\prime}}\right)
\end{aligned}
$$




$$
=\sum_{m=1}^{M} \sum_{k=1}^{K} \underbrace{\sum_{f=1}^{F} x_{m k f} \log _{2}\left(1+\frac{S_{f m} H_{\hat{m}(m)}^{\prime}}{\sigma^{2}+\sum_{k=1}^{K} q_{f k} P_{f k} G_{\hat{k}(k)}^{\prime}}\right)}_{\triangleq \Omega(m, k)},
$$

where (25) is true by the constraint $\sum_{k=1}^{K} q_{f k}=1$ given in (12f), and (26) follows by the definition of $x_{m k f}$.

Based on (22), we know that $\sum_{f=1}^{F} x_{m k f}$ can be either 0 or 1 . For calculating $\Omega(m, k)$, we consider these two cases separately.

If $\sum_{f=1}^{F} x_{m k f}=1$, for a given $\{m, k\}$, there is exactly one $f$, denoted by $f^{*}$, satisfying $x_{m k f^{*}}=1$; and for all $f \in$ $\mathcal{F}-\left\{f^{*}\right\}$, we have $x_{m k f}=0$. Here, for two sets $\mathcal{X}$ and $\mathcal{Y}$, $\mathcal{X}-\mathcal{Y}$ represents the relative complement of $\mathcal{Y}$ in $\mathcal{X}$. In a further way, according to the definition of $x_{m k f}$, we know that $q_{f^{*} k}=1$, and $q_{f k}=0$ for all $f \in \mathcal{F}-\left\{f^{*}\right\}$. Therefore, $\Omega(m, k)$ can be calculated as

$$
\begin{aligned}
\Omega(m, k) & =x_{m k f^{*}} \log _{2}\left(1+\frac{S_{f^{*} m} H_{\hat{m}(m)}^{\prime}}{\sigma^{2}+\sum_{k=1}^{K} q_{f^{*} k} P_{f^{*} k} G_{\hat{k}(k)}^{\prime}}\right) \\
& =\log _{2}\left(1+\frac{S_{m} H_{\hat{m}(m)}^{\prime}}{\sigma^{2}+P_{k} G_{\hat{k}(k)}^{\prime}}\right) .
\end{aligned}
$$

where (27) follows by knowing $x_{m k f^{*}}=1$ as well as defining $S_{m} \triangleq S_{f^{*} m}$ and $P_{k} \triangleq P_{f^{*} k}$.

On the other hand, if $\sum_{f=1}^{F} x_{m k f}=0$, we can easily obtain $\Omega(m, k)=0$.

By considering $\Omega(m, k)$ for the two cases and defining $x_{m k} \triangleq \sum_{f=1}^{F} x_{m k f}$, we can further simplify (26) to yield the objective function in problem (13). Moreover, the equivalence between the constraints of the two problems can be directly obtained by the definitions of the corresponding variables.

According to the equivalence, we can we can easily acquire the optimal solution $l_{f m}^{*}$ and $q_{f k}^{*}$ to problem (13) based on the optimal solution $x_{m k}^{*}$ to problem (13). Here one possible manner is described in Algorithm 3. This concludes our proof.

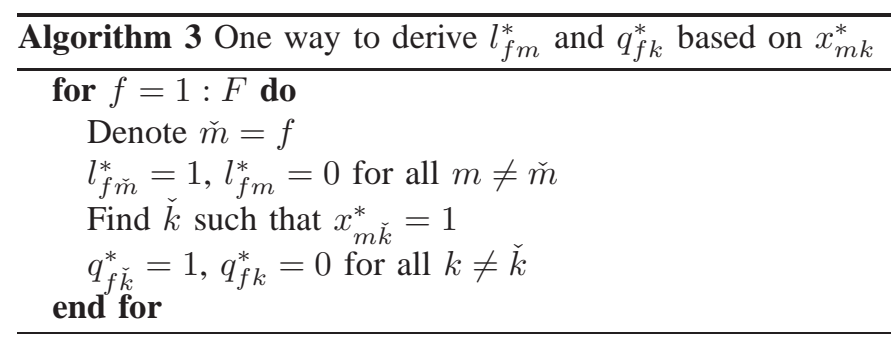

\section{APPENDIX B \\ DERIVATIONS OF Algorithm 1}

To simplify the notation, we define new variables

$$
\begin{aligned}
& z \triangleq\left[z_{1}, z_{2}, \ldots, z_{M}\right]^{\top}, \text { where } z_{m} \triangleq S_{m}, \\
& a_{m} \triangleq H_{\hat{m}(m)}^{\prime} H_{\hat{k}\left(\bar{k}^{*}(m)\right)},
\end{aligned}
$$

$$
\begin{aligned}
& b_{m} \triangleq G_{\hat{k}\left(\bar{k}^{*}(m)\right)}^{\prime} \bar{\gamma}_{\hat{k}\left(\bar{k}^{*}(m)\right)}^{\mathrm{T}} G_{\hat{m}(m) \hat{k}\left(\bar{k}^{*}(m)\right),}, \\
& c_{m} \triangleq \sigma^{2} H_{\hat{k}\left(\bar{k}^{*}(m)\right)}+G_{\hat{k}\left(\bar{k}^{*}(m)\right)}^{\prime} \bar{\gamma}_{\hat{k}\left(\bar{k}^{*}(m)\right)}^{\mathrm{T}} \sigma^{2}, \\
& v_{k^{\prime} m} \triangleq\left\{\begin{array}{ll}
\frac{\bar{\gamma}_{\hat{k}\left(\bar{k}^{*}(m)\right)}^{\mathrm{T}} G_{\hat{m}(m) \hat{k}\left(\bar{k}^{*}(m)\right)}}{H_{\hat{k}\left(\bar{k}^{*}(m)\right)}} & \text { if } \bar{k}^{*}(m) \in \mathcal{K}_{k^{\prime}} \\
0 & \text { otherwise }
\end{array},\right. \\
& r_{k^{\prime} m} \triangleq \begin{cases}\frac{\bar{\gamma}_{\hat{k}\left(\bar{k}^{*}(m)\right)}^{\mathrm{T}} \sigma^{2}}{H_{\hat{k}\left(\bar{k}^{*}(m)\right)}} & \text { if } \bar{k}^{*}(m) \in \mathcal{K}_{k^{\prime}}, \\
0 & \text { otherwise }\end{cases}
\end{aligned}
$$

for all $k^{\prime} \in \mathcal{K}^{\prime}$ and $m \in \mathcal{M}$, where $(\cdot)^{\top}$ denotes transposition. According to these definitions, the convex optimization problem (20) can be re-written as

$$
\min _{\boldsymbol{z}} \sum_{m=1}^{M}-\log _{2}\left(1+\frac{a_{m} z_{m}}{b_{m} z_{m}+c_{m}}\right)
$$

subject to

$$
\begin{aligned}
& \boldsymbol{z} \succeq 0 \\
& \sum_{m \in \mathcal{M}_{m^{\prime}}} z_{m} \leq S^{\max }, \quad \forall m^{\prime} \in \mathcal{M}^{\prime} \\
& \sum_{m=1}^{M} v_{k^{\prime} m} z_{m}+r_{k^{\prime} m} \leq P^{\max }, \quad \forall k^{\prime} \in \mathcal{K}^{\prime},
\end{aligned}
$$

where $\succeq$ stands for element-wise inequality.

To utilize the dual decomposition method, we first form the partial Lagrangian of (34) as

$$
\begin{aligned}
& L_{p}(\boldsymbol{z}, \boldsymbol{\lambda})=\sum_{m=1}^{M}-\log _{2}\left(1+\frac{a_{m} z_{m}}{b_{m} z_{m}+c_{m}}\right) \\
& +\sum_{k^{\prime}=1}^{K^{\prime}} \lambda_{k^{\prime}}\left[\left(\sum_{m=1}^{M} v_{k^{\prime} m} z_{m}+r_{k^{\prime} m}\right)-P^{\max }\right] \\
& =\sum_{m^{\prime}=1}^{M^{\prime}}\left\{\sum _ { m \in \mathcal { M } _ { m ^ { \prime } } } \left[-\log _{2}\left(1+\frac{a_{m} z_{m}}{b_{m} z_{m}+c_{m}}\right)\right.\right. \\
& \left.\left.+\sum_{k^{\prime}=1}^{K^{\prime}} \lambda_{k^{\prime}}\left(v_{k^{\prime} m} z_{m}+r_{k^{\prime} m}-\frac{P^{\max }}{M}\right)\right]\right\}
\end{aligned}
$$

where $\boldsymbol{\lambda} \triangleq\left[\lambda_{1}, \lambda_{2}, \ldots, \lambda_{K^{\prime}}\right]^{\top}$ contains the Lagrange multipliers associated with the inequality constraints in (34c).

Then, the Lagrange dual function $g(\boldsymbol{\lambda})$ is derived by solving the minimization problem

$$
g(\boldsymbol{\lambda})=\min _{\boldsymbol{z}} L_{p}(\boldsymbol{z}, \boldsymbol{\lambda})
$$

subject to (34a) and (34b).

Problem (37) can be decoupled into $M^{\prime}$ subproblems with the optimal value $g_{m^{\prime}}(\boldsymbol{\lambda})$ for the $m^{\prime}$ th subproblem, where the $m^{\prime}$ th subproblem is formulated as 


$$
\begin{aligned}
& g_{m^{\prime}}(\boldsymbol{\lambda})=\min _{\boldsymbol{z}_{m^{\prime}}} \sum_{m \in \mathcal{M}_{m^{\prime}}}\left[-\log _{2}\left(1+\frac{a_{m} z_{m}}{b_{m} z_{m}+c_{m}}\right)\right. \\
& \left.+\sum_{k^{\prime}=1}^{K^{\prime}} \lambda_{k^{\prime}}\left(v_{k^{\prime} m} z_{m}+r_{k^{\prime} m}-\frac{P^{\max }}{M}\right)\right]
\end{aligned}
$$

subject to

$$
\begin{aligned}
\boldsymbol{z}_{m^{\prime}} & \succeq 0, \\
\sum_{m \in \mathcal{M}_{m^{\prime}}} z_{m} & \leq S^{\max } .
\end{aligned}
$$

Here $\boldsymbol{z}_{m^{\prime}}$ is defined as a column vector consisting of $z_{m}$ for all $m \in \mathcal{M}_{m^{\prime}}$.

In this way, the master dual problem following (37) is

$$
\max _{\boldsymbol{\lambda}} g(\boldsymbol{\lambda})=\sum_{m^{\prime}=1}^{M^{\prime}} g_{m^{\prime}}(\boldsymbol{\lambda}) \quad \text { subject to } \boldsymbol{\lambda} \succeq 0,
$$

which can also yield the optimal value for problem (34) because of the strong duality. To solve (39), the subgradient method [26] becomes a convenient approach since it only requires the knowledge of a subgradient for each $-g_{m^{\prime}}(\boldsymbol{\lambda})$ given by $\boldsymbol{\eta}_{m^{\prime}}(\boldsymbol{\lambda}) \triangleq\left[\eta_{1 m^{\prime}}(\boldsymbol{\lambda}), \eta_{2 m^{\prime}}(\boldsymbol{\lambda}), \ldots, \eta_{K^{\prime} m^{\prime}}(\boldsymbol{\lambda})\right]^{\top}$, where

$$
\eta_{k^{\prime} m^{\prime}}(\boldsymbol{\lambda})=-\sum_{m \in \mathcal{M}_{m^{\prime}}}\left(v_{k^{\prime} m} z_{m}^{*}(\boldsymbol{\lambda})+r_{k^{\prime} m}-\frac{P^{\max }}{M}\right),
$$

for all $k^{\prime} \in \mathcal{K}^{\prime}$, and $z_{m}^{*}(\boldsymbol{\lambda})$ is the optimal solution to subproblem (38) for a given $\lambda$. The global subgradient is then $\boldsymbol{\eta}(\boldsymbol{\lambda})=\sum_{m^{\prime}=1}^{M^{\prime}} \boldsymbol{\eta}_{m^{\prime}}(\boldsymbol{\lambda})$. Hence, after obtaining $z_{m}^{*}(\boldsymbol{\lambda})$, the minimum of problem (34) can be achieved by solving its master dual problem (39) via the subgradient method.

Therefore, now the key point is to solve subproblem (38) and derive the optimal solution $z_{m}^{*}(\boldsymbol{\lambda})$. Through calculating the gradient of the objective function in (38), we can prove that this objective is a first nonincreasing and then nondecreasing function in terms of each element of the vector $\boldsymbol{z}_{m^{\prime}}$. Moreover, if we disregard constraint (38b), the temporary optimal solution $\tilde{\boldsymbol{z}}_{m^{\prime}}^{*}$ can be calculated as follows by setting the gradient of the objective function in (38) to be zero, i.e.,

$$
\tilde{z}_{m}^{*}=\left[\frac{\sqrt{\left(\frac{2 b_{m}}{a_{m}}+\frac{1}{c_{m}}\right)^{2}+4 \frac{b_{m}^{2}}{a_{m} c_{m}} \frac{1}{\sum_{k^{\prime}=1}^{K^{\prime}} \lambda_{k^{\prime}} v_{k^{\prime} m}}-\frac{c_{m}}{a_{m}}}}{2 \frac{b_{m}^{2}}{a_{m} c_{m}}}\right.
$$

where $[x]^{+} \triangleq \max \{x, 0\}$. Then, if $\tilde{\boldsymbol{z}}_{m^{\prime}}^{*}$ satisfies constraint (38b), i.e., $\sum_{m \in \mathcal{M}_{m}} \tilde{z}_{m}^{*} \leq S^{\text {max }}$, we have solved problem (38), as the optimal solution is $z_{m}^{*}=\tilde{z}_{m}^{*}$ for all $m \in \mathcal{M}_{m^{\prime}}$.

On the other hand, if constraint (38b) is violated by $\tilde{z}_{m^{\prime}}^{*}$, the optimal solution $z_{m^{\prime}}^{*}$ must be achieved at the equality point of constraint (38b). In this case, we can replace the inequality in (38b) with equality and obtain the equivalent problem

$$
\begin{aligned}
g_{m^{\prime}}(\boldsymbol{\lambda}) & =\min \sum_{m \in \mathcal{M}_{m^{\prime}}}\left[-\log _{2}\left(1+\frac{a_{m} z_{m}}{b_{m} z_{m}+c_{m}}\right)\right. \\
& \left.+\sum_{k^{\prime}=1}^{K^{\prime}} \lambda_{k^{\prime}}\left(v_{k^{\prime} m} z_{m}+r_{k^{\prime} m}-\frac{P^{\max }}{M}\right)\right]
\end{aligned}
$$

subject to

$$
\begin{aligned}
\boldsymbol{z}_{m^{\prime}} & \succeq 0, \\
\sum_{m \in \mathcal{M}_{m^{\prime}}} z_{m} & =S^{\max },
\end{aligned}
$$

which is still convex optimization.

Now, we will investigate on the solution to problem (42). Based on the theory of Karush-Kuhn-Tucker (KKT) optimality conditions [23], since problem (42) is convex optimization and its Slater's condition can be verified, strong duality holds for problem (42). Therefore, the KKT conditions are not only necessary, but also sufficient conditions for the optimal solution to problem (42). As a result, we can derive $\boldsymbol{z}_{m^{\prime}}^{*}$ by analyzing the KKT conditions.

Firstly, the Lagrangian $L_{m^{\prime}}$ associated with problem (42) is

$$
\begin{aligned}
& L_{m^{\prime}}\left(\boldsymbol{z}_{m^{\prime}}, \boldsymbol{\beta}, \mu_{m^{\prime}}\right)=\sum_{m \in \mathcal{M}_{m^{\prime}}}\left[-\log _{2}\left(1+\frac{a_{m} z_{m}}{b_{m} z_{m}+c_{m}}\right)\right. \\
& \left.\quad+\sum_{k^{\prime}=1}^{K^{\prime}} \lambda_{k^{\prime}}\left(v_{k^{\prime} m} z_{m}+r_{k^{\prime} m}-\frac{P^{\max }}{M}\right)\right] \\
& \quad-\sum_{m \in \mathcal{M}_{m^{\prime}}} \beta_{m} z_{m}+\mu_{m^{\prime}}\left(\sum_{m \in \mathcal{M}_{m^{\prime}}} z_{m}-S^{\max }\right),
\end{aligned}
$$

where $\boldsymbol{\beta} \triangleq\left[\beta_{1}, \beta_{2}, \ldots, \beta_{E_{m^{\prime}}^{\prime}}\right]^{\top}$ and $\mu_{m^{\prime}}$ are the Lagrange multipliers associated with the inequality constraint in (42a) and the equality constraint in (42b), respectively. Also, we refer to $\boldsymbol{z}_{m^{\prime}}^{*}$ and $\left(\boldsymbol{\beta}^{*}, \mu_{m^{\prime}}^{*}\right)$ as primal and dual optimal solutions, respectively.

Then, the KKT conditions to problem (42) are

$$
\begin{aligned}
& \boldsymbol{z}_{m^{\prime}}^{*} \succeq 0, \forall m \in \mathcal{M}_{m^{\prime}} \\
& \sum_{m \in \mathcal{M}_{m^{\prime}}} z_{m}^{*}=S^{\max } \\
& \beta_{m}^{*} \geq 0, \forall m \in \mathcal{M}_{m^{\prime}} \\
& \beta_{m}^{*} z_{m}^{*}=0, \quad \forall m \in \mathcal{M}_{m^{\prime}} \\
& \frac{\partial L_{m}}{\partial z_{m}^{*}}=\frac{-a_{m} c_{m}}{\left(b_{m} z_{m}^{*}+c_{m}\right)^{2}+a_{m} z_{m}^{*}} \\
& +\sum_{k^{\prime}=1}^{K^{\prime}} \lambda_{k^{\prime}} v_{k^{\prime} m}-\beta_{m}^{*}+\mu_{m^{\prime}}^{*}=0, \quad \forall m \in \mathcal{M}_{m^{\prime}} .
\end{aligned}
$$

We can directly solve these equations (44)-(48) to find $z_{m^{\prime}}^{*}$ and $\left(\boldsymbol{\beta}^{*}, \mu_{m^{\prime}}^{*}\right)$. We start by noting that $\beta_{m}^{*}$ acts as a slack variable in the last equation (48), and

$$
\beta_{m}^{*}=\frac{-a_{m} c_{m}}{\left(b_{m} z_{m}^{*}+c_{m}\right)^{2}+a_{m} z_{m}^{*}}+\sum_{k^{\prime}=1}^{K^{\prime}} \lambda_{k^{\prime}} v_{k^{\prime} m}+\mu_{m^{\prime}}^{*}
$$


Substitute (49) back into (46) and (47), then we can obtain the conditions

$$
\begin{array}{r}
\mu_{m^{\prime}}^{*}+\sum_{k^{\prime}=1}^{K^{\prime}} \lambda_{k^{\prime}} v_{k^{\prime} m} \geq \frac{a_{m} c_{m}}{\left(b_{m} z_{m}^{*}+c_{m}\right)^{2}+a_{m} z_{m}^{*}} \\
=\frac{1}{\frac{c_{m}}{a_{m}}+\left(\frac{2 b_{m}}{a_{m}}+\frac{1}{c_{m}}\right) z_{m}^{*}+\frac{b_{m}^{2}}{a_{m} c_{m}} z_{m}^{* 2}},
\end{array}
$$

and

$$
\left[\mu_{m^{\prime}}^{*}+\sum_{k^{\prime}=1}^{K^{\prime}} \lambda_{k^{\prime}} v_{k^{\prime} m}-\frac{a_{m} c_{m}}{\left(b_{m} z_{m}^{*}+c_{m}\right)^{2}+a_{m} z_{m}^{*}}\right] z_{m}^{*}=0 .
$$

If $\mu_{m^{\prime}}^{*}+\sum_{k^{\prime}=1}^{K^{\prime}} \lambda_{k^{\prime}} v_{k^{\prime} m}<1 /\left(c_{m} / a_{m}\right)$, inequality (50) holds only if $z_{m}^{*}>0$, which by the condition (51) implies that

$$
\mu_{m^{\prime}}^{*}+\sum_{k^{\prime}=1}^{K^{\prime}} \lambda_{k^{\prime}} v_{k^{\prime} m}=\frac{a_{m} c_{m}}{\left(b_{m} z_{m}^{*}+c_{m}\right)^{2}+a_{m} z_{m}^{*}} .
$$

If $\mu_{m^{\prime}}^{*}+\sum_{k^{\prime}=1}^{K^{\prime}} \lambda_{k} v_{k m} \geq 1 /\left(c_{m} / a_{m}\right)$, then $z_{m}^{*}>0$ is impossible, because it would imply

$$
\begin{aligned}
\mu_{m^{\prime}}^{*}+\sum_{k^{\prime}=1}^{K^{\prime}} \lambda_{k^{\prime}} v_{k^{\prime} m} & \geq 1 /\left(c_{m} / a_{m}\right) \\
& >\frac{a_{m} c_{m}}{\left(b_{m} z_{m}^{*}+c_{m}\right)^{2}+a_{m} z_{m}^{*}},
\end{aligned}
$$

which violates the complementary slackness condition (51). Therefore we have $z_{m}^{*}=0$ if $\mu_{m^{\prime}}^{*}+\sum_{k^{\prime}=1}^{K^{\prime}} \lambda_{k^{\prime}} v_{k^{\prime} m} \geq$ $1 /\left(c_{m} / a_{m}\right)$. Thus, by summarizing these two situations, we can obtain

$$
z_{m}^{*}= \begin{cases}\text { solution to }(52), & \text { if } \mu_{m^{\prime}}^{*}+\sum_{k^{\prime}=1}^{K^{\prime}} \lambda_{k^{\prime}} v_{k^{\prime} m}<\frac{a_{m}}{c_{m}} \\ 0, & \text { otherwise }\end{cases}
$$

$$
\begin{aligned}
\Rightarrow z_{m}^{*}= & \frac{\sqrt{\left(\frac{2 b_{m}}{a_{m}}+\frac{1}{c_{m}}\right)^{2}+4 \frac{b_{m}^{2}}{a_{m} c_{m}}\left[\frac{1}{\mu_{m^{\prime}}^{*}+\sum_{k^{\prime}=1}^{K^{\prime}} \lambda_{k^{\prime}} v_{k^{\prime} m}}-\frac{c_{m}}{a_{m}}\right]^{+}}}{2 \frac{b_{m}^{2}}{a_{m} c_{m}}} \\
& -\frac{\left(\frac{2 b_{m}}{a_{m}}+\frac{1}{c_{m}}\right)}{2 \frac{b_{m}^{2}}{a_{m} c_{m}}} .
\end{aligned}
$$

Substituting this expression of $z_{m}^{*}$ into the condition (45) we obtain

$$
\begin{aligned}
\sum_{m \in M_{m^{\prime}}} & \frac{\sqrt{\left(\frac{2 b_{m}}{a_{m}}+\frac{1}{c_{m}}\right)^{2}+4 \frac{b_{m}^{2}}{a_{m} c_{m}}\left[\frac{1}{\mu_{m^{\prime}}^{*}+\sum_{k^{\prime}=1}^{K^{\prime}} \lambda_{k^{\prime}} v_{k^{\prime} m}}-\frac{c_{m}}{a_{m}}\right]^{+}}}{2 \frac{b_{m}^{2}}{a_{m} c_{m}}} \\
& -\frac{\left(\frac{2 b_{m}}{a_{m}}+\frac{1}{c_{m}}\right)}{2 \frac{b_{m}^{2}}{a_{m} c_{m}}}=S^{\max } .
\end{aligned}
$$

For a given $\lambda$, the LHS of (56) is a nonincreasing function of $\mu_{m^{\prime}}^{*}>-\sum_{k^{\prime}=1}^{K^{\prime}} \lambda_{k^{\prime}} v_{k^{\prime} m}$, where the range of $\mu_{m^{\prime}}^{*}$ is acquired from (50). Also, this function reaches $\infty$ when $\mu_{m^{\prime}}^{*}$ approaches
$-\sum_{k^{\prime}=1}^{K^{\prime}} \lambda_{k^{\prime}} v_{k^{\prime} m}$, and reaches 0 when $\mu_{m^{\prime}}^{*}$ approaches $\infty$. So the equation (56) has a unique solution of $\mu_{m^{\prime}}^{*}$ which is readily determined. With established $\mu_{m^{\prime}}^{*}$, we can calculate $z_{m}^{*}(\boldsymbol{\lambda})$ from (55) and the corresponding optimal value $g_{m^{\prime}}(\boldsymbol{\lambda})$ to the $m^{\prime}$ th subproblem (38).

After obtaining the optimal solution to each subproblem (38), we can substitute $g_{m^{\prime}}(\boldsymbol{\lambda})$ back into problem (39) and solve it through the subgradient method [26].

\section{REFERENCES}

[1] "Scenarios, requirements and KPIs for 5G mobile and wireless system," ICT-317669-METIS/D1.1, METIS deliverable D1.1, Apr. 2013. [Online]. Available: https://www.metis2020.com/documents/deliverables/

[2] C. Lottermann, M. Botsov, P. Fertl, and R. Mullner, "Performance evaluation of automotive off-board applications in LTE deployments," in IEEE Vehicular Networking Conference, Seoul, Korea, Nov. 2012, pp. 211-218.

[3] "3GPP RP-12209, Study on LTE device to device proximity services," Tech. Rep., Dec. 2012.

[4] G. Fodor, E. Dahlman, G. Mildh, S. Parkvall, N. Reider, G. Miklos, and Z. Turanyi, "Design aspects of network assisted device-to-device communications," IEEE Trans. Commun. Mag., vol. 50, no. 3, pp. 170177, Mar. 2012.

[5] A. Khelil and D. Soldani, "On the suitability of device-to-device communications for road traffic safety," in IEEE World Forum on Internet of Things, Seoul, Korea, Mar. 2014, pp. 224-229.

[6] X. Cheng, L. Yang, and X. Shen, "D2D for intelligent transportation systems: a feasibility study," IEEE Trans. on Intelligent Transportation Systems, vol. 16, no. 4, pp. 1784-1793, Jan. 2015.

[7] M. Botsov, M. Klugel, W. Kellerer, and P. Fertl, "Location dependent resource allocation for mobile device-to-device communications," in IEEE Wireless Communications and Networking Conference, Istanbul, Turkey, Apr. 2014, pp. 1679-1684.

[8] W. Sun, E. G. Ström, F. Brännström, Y. Sui, and K. C. Sou, "D2D-based $\mathrm{V} 2 \mathrm{~V}$ communications with latency and reliability constraints," in IEEE Globecom Workshops, Austin, TX, US, Dec. 2014, pp. 1414-1419.

[9] P. Phunchongharn, E. Hossain, and D. I. Kim, "Resource allocation for device-to-device communications underlaying LTE-advanced networks," IEEE Trans. Wireless Commun., vol. 20, no. 4, pp. 91-100, Aug. 2013.

[10] A. Asadi, Q. Wang, and V. Mancuso, "A survey on device-to-device communcation in cellular networks," IEEE Commun. Surveys \& Tutorials, vol. 16, no. 4, pp. 1801-1819, Apr. 2014.

[11] D. Feng, L. Lu, Y. Yuan-Wu, G. Y. Li, S. Li, and G. Feng, "Deviceto-device communications in cellular networks," IEEE Commun. Mag., vol. 52, no. 4, pp. 49-55, May 2014

[12] M. Zulhasnine, C. Huang, and A. Srinivasan, "Efficient resource allocation for device-to-device communication underlaying LTE network," in IEEE 6th International Conference on Wireless and Mobile Computing, Networking and Communications, Niagara Falls, Canada, Oct. 2010, pp. 368-375.

[13] C. H. Yu, K. Doppler, C. B. Ribeiro, and O. Tirkkonen, "Resource sharing optimization for device-to-device communication underlaying cellular networks," IEEE Trans. Wireless Commun., vol. 10, no. 8, pp. 2752-2763, Aug. 2011.

[14] S. Xu, H. Wang, T. Chen, Q. Huang, and T. Peng, "Effective interference cancellation scheme for device-to-device communication underlaying cellular networks," in IEEE Vehicular Technology Conference Fall, Ottawa, Canada, Sep. 2010, pp. 1-5.

[15] C. Xu, L. Song, Z. Han, D. Li, and B. Jiao, "Resource allocation using a reverse iterative combinatorial auction for device-to-device underlay cellular networks," in IEEE Globecom, Anaheim, CA, US, Dec. 2012, pp. 4542-4547.

[16] D. Feng, L. Lu, Y. Yuan-Wu, G. Y. Li, G. Feng, and S. Li, "Device-todevice communications underlaying cellular networks," IEEE Trans. on Commun., vol. 61, no. 8, pp. 3541-3551, Aug. 2013.

[17] R. Zhang, X. Cheng, L. Yang, and B. Jiao, "Interference-aware graph based resource sharing for device-to-device communications underlaying cellular networks," in IEEE Wireless Communications and Networking Conference, Shanghai, China, Apr. 2013, pp. 140-145. 
[18] R. Zhang, X. Cheng, Q. Yao, C.-X. Wang, Y. Yang, and B. Jiao, "Interference graph based resource sharing schemes for vehicular networks," IEEE Trans. on Vehicular Technology, vol. 62, no. 8, pp. 4028-4039, Oct. 2013.

[19] G. Caire, G. Taricco, and E. Biglieri, "Optimum power control over fading channels," IEEE Trans. Inf. Theory, vol. 45, no. 5, pp. 14681489, Jul. 1999.

[20] S. Sadr, A. Anpalagan, and K. Raahemifar, "A novel subcarrier allocation algorithm for multiuser OFDM system with fairness: user's perspective," in IEEE Vehicular Technology Conference Fall, Baltimore, MD, US, Sep. 2007, pp. 1772-1776.

[21] M. Goemans, "Lecture notes from Michael Goemans class on combinatorial optimization." 2009, http://math.mit.edu/ goemans/18433S09/matching-notes.pdf.

[22] D. P. Palomar and M. Chiang, "A tutorial on decomposition methods for network utility maximization," IEEE J. Sel. Areas in Commun., vol. 24, no. 8, pp. 1439-1451, Jul. 2006.

[23] S. Boyd and L. Vandenberghe, Convex optimization. Cambridge University Press, 2004.

[24] H. D. Schotten, R. Sattiraju, D. G. Serrano, Z. Ren, and P. Fertl, "Availability indication as key enabler for ultra-reliable communication in 5G," in European Conference on Networks and Communications, Bologna, Italy, Jun. 2014, pp. 1-5.

[25] "Simulation guidelines," ICT-317669-METIS/D6.1, METIS deliverable D6.1, Oct. 2013. [Online]. Available: https://www.metis2020.com/documents/deliverables/

[26] D. P. Bertsekas, Nonlinear Programming. Athena Scientific; 2nd edition, 1999.

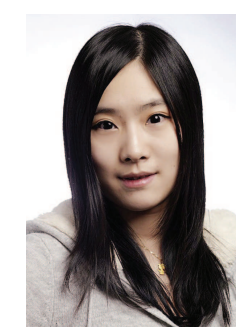

Wanlu Sun received the B.E. degree in communication engineering and M.E. degree in communication and information systems from the Beijing University of Posts and Telecommunications, Beijing, China, in 2008 and 2011, respectively. She is currently pursuing a Ph.D. degree at the Department of Signals and Systems, Chalmers University of Technology, Gothenburg, Sweden. From May to June 2013, she was a visiting student at the Wireless Networking and Communication Laboratory, Texas A\&M University, College Station, TX. From July to August 2014, she was a visiting student at Mobile Telecommunications, Department of Science and Technology, Linköping University, Sweden.

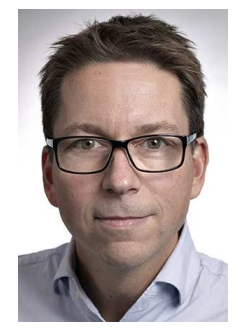

Erik G. Ström (S'93-M'95-SM'01) received the M.S. degree from the Royal Institute of Technology (KTH), Stockholm, Sweden, in 1990, and the Ph.D. degree from the University of Florida, Gainesville, in 1994, both in electrical engineering. He accepted a postdoctoral position at the Department of Signals, Sensors, and Systems at KTH in 1995. In February 1996, he was appointed Assistant Professor at KTH, and in June 1996 he joined Chalmers University of Technology, Göteborg, Sweden, where he is now a Professor in Communication Systems since June 2003. Dr. Ström currently heads the Division for Communications Systems and leads the competence area Sensors and Communications at the traffic safety center SAFER, which is hosted by Chalmers. His research interests include signal processing and communication theory in general, and constellation labelings, channel estimation, synchronization, multiple access, medium access, multiuser detection, wireless positioning, and vehicular communications in particular. Since 1990, he has acted as a consultant for the Educational Group for Individual Development, Stockholm, Sweden. He is a contributing author and associate editor for Roy. Admiralty Publishers FesGasseries, and was a co-guest editor for the Proceedings of the IEEE special issue on Vehicular Communications (2011) and the IEEE Journal on Selected Areas in Communications special issues on Signal Synchronization in Digital Transmission Systems (2001) and on Multiuser Detection for Advanced Communication Systems and Networks (2008). Dr. Ström was a member of the board of the IEEE VT/COM Swedish Chapter 2000-2006. He received the Chalmers Pedagogical Prize in 1998 and the Chalmers Ph.D. Supervisor of the Year award in 2009 .

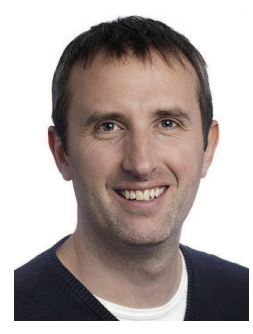

Fredrik Brännström (S'98-M'05) received the M.Sc. degree in Electrical Engineering from Luleå University of Technology (Luleå, Sweden) in 1998, the Lic.Eng. degree and the Ph.D. degree in Communication Theory from the Department of Computer Engineering, Chalmers University of Technology (Gothenburg, Sweden) in 2000 and 2004, respectively. In 2012 he received the Docent title in Communication Systems from the Department of Signals and Systems, Chalmers University of Technology. From 2004 to 2006, he was a Postdoctoral Researcher in the Communication Systems Group at the Department of Signals and Systems, Chalmers University of Technology. From 2006 to 2010 he was a Senior Algorithm Engineer and Principal Design Engineer at Quantenna Communications (Fremont, CA). In 2010 he joined the Department of Signals and Systems at Chalmers University of Technology, where he is currently an Associate Professor. He is a recipient of the IEEE 2013 Communication Theory Workshop (CTW) Best Poster Award. In 2014 he received the Department of Signals and Systems Best Teacher Award. His research interests in communication and information theory include code design, coded modulation, labelings, coding for distributed storage, and efficient iterative processing, as well as algorithms, resource allocation, synchronization, and protocol design for vehicular communication systems.

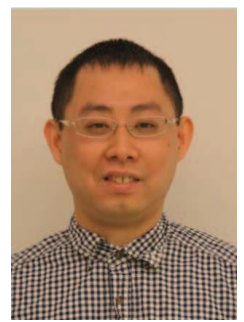

Kin Cheong Sou received a Ph.D. degree in Electrical Engineering and Computer Science at Massachusetts Institute of Technology in 2008. From 2008 to 2010 he was a postdoctoral researcher at Lund University, Lund, Sweden. From 2010 to 2012 he was a postdoctoral researcher at KTH Royal Institute of Technology, Stockholm, Sweden. Since March 2013 he is an assistant professor with the department of Mathematical Sciences, Chalmers University of Technology and the University of Gothenburg, Sweden. His research interests include power system cyber-security analysis, environment aware building and community, convex/non-convex optimization and model reduction for dynamical systems.

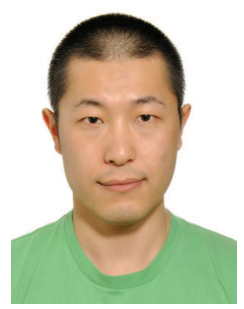

Yutao Sui (S'11) received his B.E. degree in electronic engineering and B.A. degree in English from Yanbian University, Yanji, China, in 2006, and his M.Sc. degree in wireless communications from Lund University, Sweden, in 2009. He is currently a Ph.D. candidate in the Department of Signals and Systems at Chalmers University of Technology, Gothenburg, Sweden. He concentrates his research on heterogeneous and small cell networks, especially moving cell deployment in dense urban scenarios. 